\title{
Imidazolium-linked azido-functionalized Guerbet glycosides: multifunctional surfactants for biofunctionalization of vesicles
}

\section{- Supplementary Material -}

Ean Wai Goh, ${ }^{1}$ Thorsten Heidelberg, ${ }^{1 *}$ Rusnah Syahila Duali Hussen, ${ }^{1}$ Abbas Abdulameer Salman ${ }^{1,2}$

${ }^{1}$ Chemistry Department, Faculty of Science, University of Malaya, 50603 Kuala Lumpur, Malaysia

${ }^{2}$ current affiliation: College of Pathological Analysis Technologies, Al-Bayan University, Baghdad, Iraq

heidelberg@um.edu.my 


\section{Physical Studies}

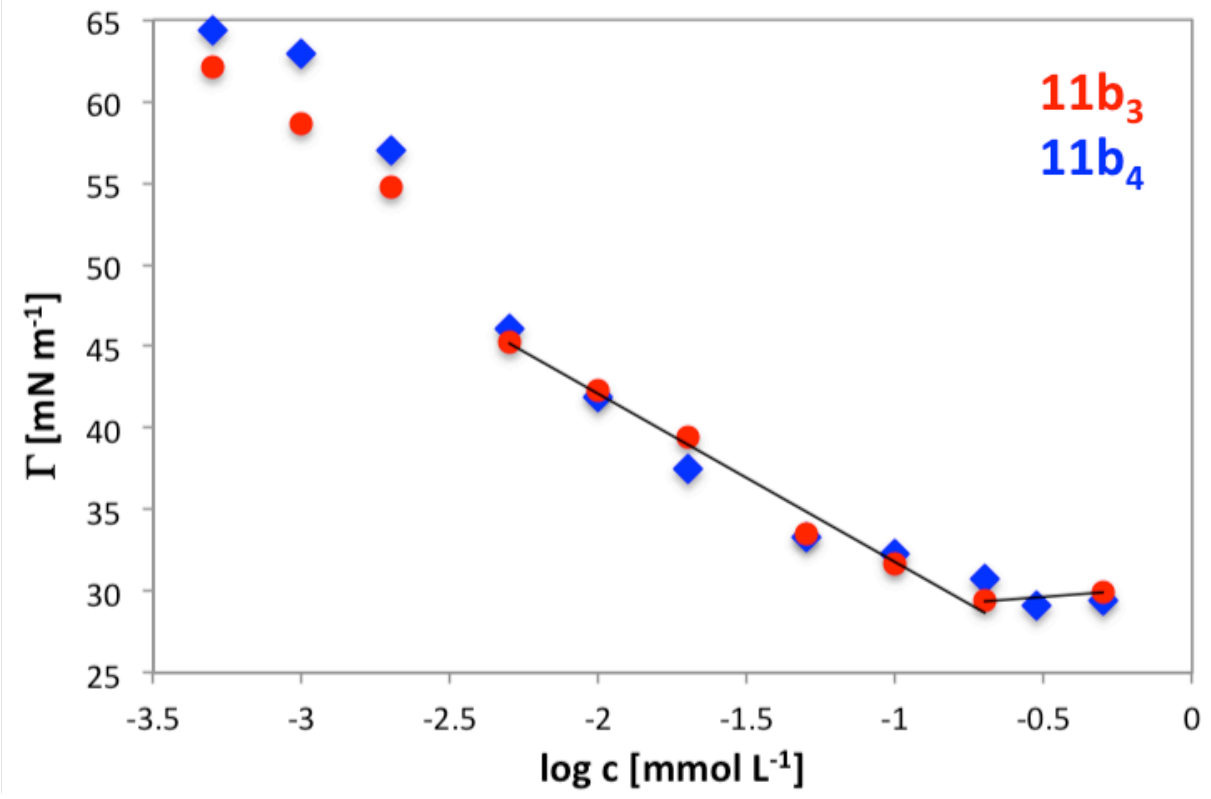

Figure S1. Surface tension behavior of $\mathbf{1 1}$

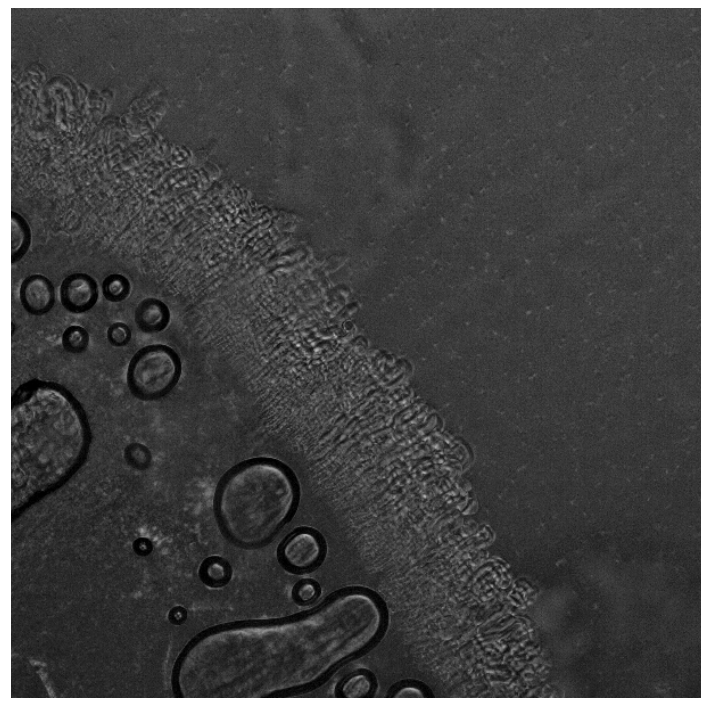

Figure S2. Contact penetration of a mixture of $\mathbf{1 2}$ and $\mathbf{1 1} \mathbf{b}_{\mathbf{3}}(10 \%)$ with water under the optical polarizing microscope; massive formation of myelin figures indicates the lamellar phase 


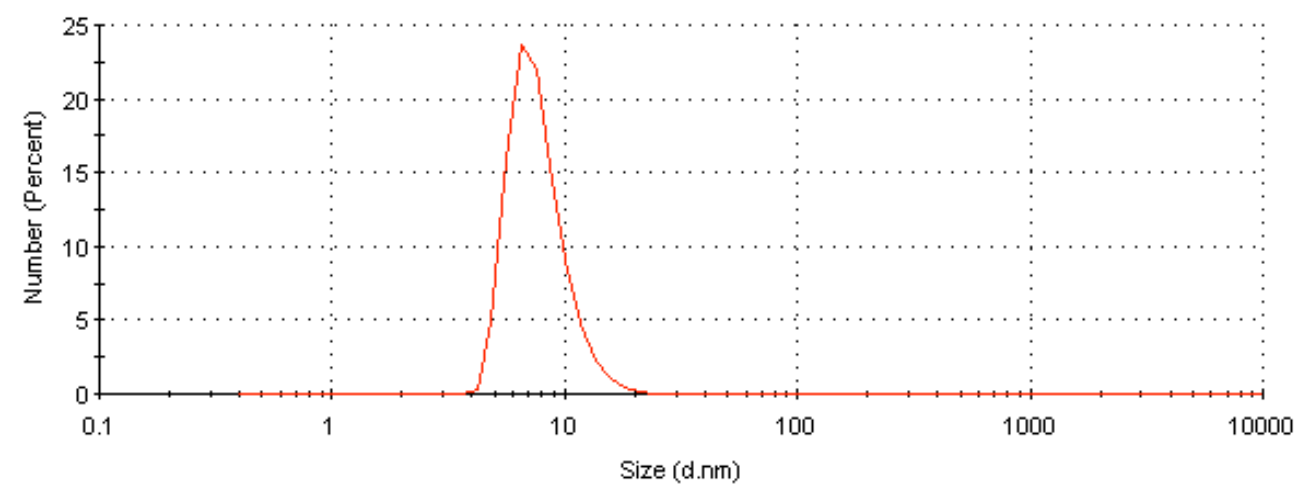

Figure S3. Vesicle size distribution for $\mathbf{1 2}$ with $5 \% \mathbf{1 1} \mathbf{b}_{\mathbf{3}}$

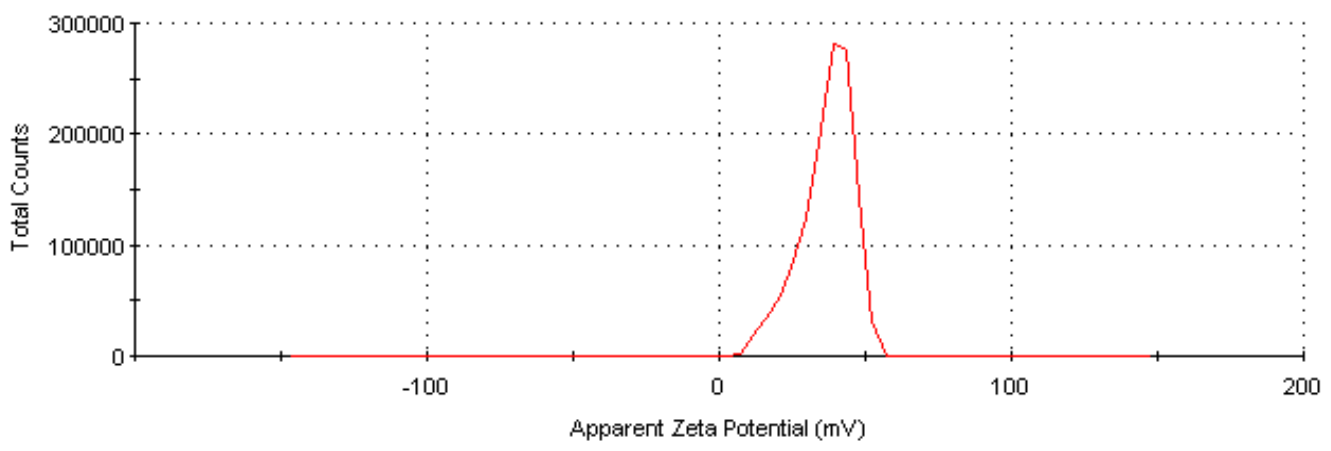

Figure S4. Zeta-potential distribution for vesicles of 12 with $5 \% \mathbf{1 1} \mathbf{b}_{3}$ 


\section{Experimental}

Compounds containing remaining impuities

2-Butyl-octyl 6-[1-(8-azido-3,6-dioxa-octyl)-imidazolium-3-yl]-6-deoxy- $\beta$-D-glucopyranoside

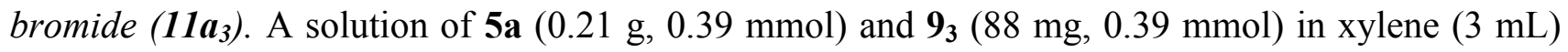
was heated to $130^{\circ} \mathrm{C}$. when TLC indicated the absence of starting material the solvent was evaporated to provide $\mathbf{1 0 a}_{3}(0.27 \mathrm{~g}, 91 \%)$ as a yellow syrup. ${ }^{1} \mathrm{H}$ NMR analysis indicated about $25-30 \%$ remaining $\mathbf{5 a}$ as impurity.

The intermediate $\mathbf{1 0 a}_{3}\left(72 \mathrm{mg}, 0.09 \mathrm{mmol}\right.$ ) was subjected to Zemplen deacetylation in $\mathrm{CH}_{3} \mathrm{OH}$ $(5 \mathrm{~mL})$ using a catalytic amount of $\mathrm{NaOMe}$. After stirring at $\mathrm{rt}$ overnight the catalyst was removed by treatment with Amberlite IR120 $\left(\mathrm{H}^{+}\right)$and the solvent was evaporated to furnish $\mathbf{1 1 \mathbf { a } _ { 3 }}(54 \mathrm{mg}, 89 \%)$ as yellow syrup. The starting material impurities form $\mathbf{1 0 a _ { 3 }}$ remained.

Peracetate 10a $:[\alpha]_{\mathrm{D}}^{25}=-20\left(\mathrm{c} 0.38, \mathrm{CHCl}_{3}\right) .{ }^{1} \mathrm{H}-\mathrm{NMR}\left(400 \mathrm{MHz}, \mathrm{CDCl}_{3}\right) \delta=10.21$ (bs, imidazole), 7.48, 7.37 (2 m, 2 H, imidazole), 5.20 (dd $\sim$, H-3), 4.87 (dd, H-2), 4.78-4.62 (m, 2 H, H-6), 4.67 (dd $\sim \mathrm{t}, \mathrm{H}-4), 4.55$ (d, H-1), 4.52 (ddd $\left.\sim \mathrm{m}_{\mathrm{c}}, \mathrm{CH}_{2} \mathrm{~N}_{\text {imidazole }}-\mathrm{A}\right), 4.44$ (ddd $\left.\sim \mathrm{m}_{\mathrm{c}}, \mathrm{CH}_{2} \mathrm{~N}_{\text {imidazole }}-\mathrm{B}\right), 4.06$ (ddd bs, H-5), $3.89\left(\mathrm{~m}_{\mathrm{c}}, 2 \mathrm{H}, \mathrm{CH}_{2} \mathrm{O}\right), 3.74-3.52\left(\mathrm{~m}, 11 \mathrm{H}, \alpha-\mathrm{CH}_{2}-\mathrm{A}, \mathrm{EG}-\mathrm{CH}_{2}\right), 3.36\left(\mathrm{t}, 2 \mathrm{H}, \mathrm{CH}_{2} \mathrm{~N}_{3}\right.$ ), $3.30\left(\mathrm{dd} \sim \mathrm{m}_{\mathrm{c}}, \alpha-\mathrm{CH}_{2}-\mathrm{B}\right), 2.22,1.97,1.93(3 \mathrm{~s}, 3 \times 3 \mathrm{H}, \mathrm{Ac}), 1.48\left(\mathrm{~m}_{\mathrm{c}}, \beta-\mathrm{CH}\right), 1.20\left(\mathrm{~m}_{\mathrm{c}}, 16 \mathrm{H}\right.$, bulk- $\mathrm{CH}_{2}$ ), $0.84\left(\mathrm{t}, 6 \mathrm{H}, \mathrm{CH}_{3}\right) ;{ }^{3} J_{1,2}=8.0,{ }^{3} J_{2,3}=9.5,{ }^{3} J_{3,4}=9.5,{ }^{3} J_{4,5}=9.5,{ }^{2} J_{6}=14.5 \mathrm{~Hz} .{ }^{13} \mathrm{C} \mathrm{NMR}$ $\left(100 \mathrm{MHz}, \mathrm{CDCl}_{3}\right) \delta=170.50,169.66,169.14(\mathrm{CO}), 138.44$ (imidazole-CHN $\left.{ }_{2}\right), 122.74,122.58$

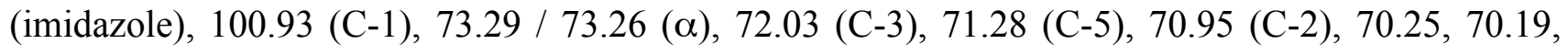
$69.84\left(\mathrm{EG}-\mathrm{CH}_{2}\right), 68.77\left(\mathrm{CH}_{2} \mathrm{O}\right), 68.22(\mathrm{C}-4), 50.55\left(\mathrm{CH}_{2} \mathrm{~N}_{3}\right), 49.90\left(\mathrm{CH}_{2} \mathrm{~N}_{\text {imidazole }}\right), 49.40(\mathrm{C}-6)$, $37.86(\beta), 31.70(\omega-2), 30.95,30.71,30.60,30.36,29.55$ / 29.53, 28.88, 28.73 (bulk- $\left.\mathrm{CH}_{2}\right), 26.67$ / $26.51(\gamma), 22.88,22.52(\omega-1), 21.34,20.40,20.35($ Ac) $), 13.95(\omega)$.

11a $a_{3}$ IR [ATR, neat] $v / \mathrm{cm}^{-1} 3370(\mathrm{OH}), 2925,2858(\mathrm{CH}), 2106\left(\mathrm{~N}_{3}\right)$. [ $\left.\alpha\right]_{\mathrm{D}}{ }^{25}=-12\left(\mathrm{c} 0.24, \mathrm{CH}_{3} \mathrm{OH}\right)$. ${ }^{1} \mathrm{H}$ NMR (400 MHz, $\mathrm{CD}_{3} \mathrm{OD}$ ): $\delta 9.00$ (s, imidazole-CHN 2 ), 7.73, 7.61 (2 s, $2 \mathrm{H}$, imidazole), 4.68 (dd,

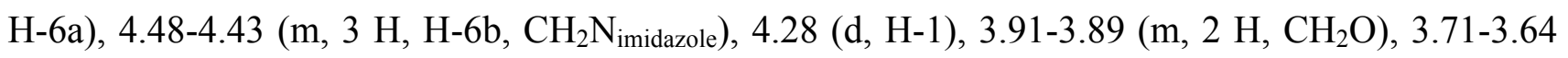
(m, 8 H, EG-CH $\left.2, \alpha-\mathrm{CH}_{2}-\mathrm{A}, \mathrm{H}-5\right), 3.43-3.36$ (m, $\left.4 \mathrm{H}, \mathrm{CH}_{2} \mathrm{~N}_{3}, \alpha-\mathrm{CH}_{2}-\mathrm{B}, \mathrm{H}-3\right), 3.17$ (dd, H-2), 3.08 (dd t, H-4), $1.60\left(\mathrm{~m}_{\mathrm{c}}, \beta-\mathrm{CH}\right), 1.32\left(\mathrm{~m}_{\mathrm{c}}, 16 \mathrm{H}\right.$, bulk- $\left.\mathrm{CH}_{2}\right), 0.93\left(\mathrm{~m}_{\mathrm{c}}, 6 \mathrm{H}, \mathrm{CH}_{3}\right) ;{ }^{3} J_{1,2}=8.0,{ }^{3} J_{2,3}=9.5$, ${ }^{3} J_{3,4}=9.5, \quad{ }^{3} J_{4,5}=9.5,{ }^{3} J_{5,6 \mathrm{a}}=2.0,{ }^{3} J_{6 \mathrm{a}, 6 \mathrm{~b}}=14.5 \mathrm{~Hz} ;{ }^{13} \mathrm{C}$ NMR $\left(100 \mathrm{MHz}, \mathrm{CD}_{3} \mathrm{OD}\right): \delta 138.7$ (imidazole-CHN 2 ), 124.7, 124.1 (imidazole), 105.0 (C-1), 77.7 (C-3), 75.1 (C-2), 74.9 (C-5), 74.29 / $74.26(\alpha), 72.5(\mathrm{C}-4), 71.6(2), 71.2\left(\mathrm{EG}^{\left.-\mathrm{CH}_{2}\right)}, 70.0\left(\mathrm{CH}_{2} \mathrm{O}\right), 51.9(\mathrm{C}-6), 51.9\left(-\mathrm{CH}_{2} \mathrm{~N}_{3}\right), 51.1\right.$ $\left(\mathrm{CH}_{2} \mathrm{~N}_{\text {imidazole }}\right), 39.64$ ( $\left.\beta\right), 33.2,32.4$ / 32.3, 32.1 / 32.0, 30.99, 30.31 / 30.25, 28.03 / 27.96, 24.3 (bulk $\mathrm{CH}_{2}$ ), $23.9(\omega-1), 14.6(\omega)$. HRMS (ESI): Calc. for [M-Br] $\left[\mathrm{C}_{27} \mathrm{H}_{50} \mathrm{~N}_{5} \mathrm{O}_{7}\right]^{+}$556.3710, 557.3744 (30\%); found $556.3727,557.3757(34 \%)$. 
2-Hexyl-decyl 6-[1-(5-azido-3-oxa-pentyl)-imidazolium-3yl]-6-deoxy- $\beta$-D-glucopyranoside bromide (11 $\left.\mathbf{b}_{2}\right)$. A solution of $\mathbf{5 b}(0.12 \mathrm{~g}, 0.20 \mathrm{mmol})$ and $\mathbf{9} \mathbf{2}(37 \mathrm{mg}, 0.20 \mathrm{mmol})$ in xylene $(2 \mathrm{~mL})$ was heated to $130{ }^{\circ} \mathrm{C}$. when TLC indicated the absence of starting material the solvent was evaporated to provide $\mathbf{1 0 b}_{2}(0.14 \mathrm{~g}, 89 \%)$ as a yellow syrup. ${ }^{1} \mathrm{H}$ NMR analysis indicated about $20-25 \%$ remaining $\mathbf{5 b}$ as impurity.

The intermediate $\mathbf{1 0 b}_{2}(0.12 \mathrm{~g}, 0.15 \mathrm{mmol})$ was subjected to Zemplen deacetylation in $\mathrm{CH}_{3} \mathrm{OH}$ $(5 \mathrm{~mL})$ using a catalytic amount of $\mathrm{NaOMe}$. After stirring at rt overnight the catalyst was removed by treatment with Amberlite IR120 $\left(\mathrm{H}^{+}\right)$and the solvent was evaporated to furnish $\mathbf{1 1} \mathbf{b}_{\mathbf{2}}(90 \mathrm{mg}, 92 \%)$ as yellow syrup. The starting material impurities form $\mathbf{1 0 b _ { 2 }}$ remained.

Peracetate 10b $\mathbf{b}_{2}:[\mathrm{a}]_{\mathrm{D}}{ }^{25}=-20\left(\mathrm{c} 0.1, \mathrm{CHCl}_{3}\right) .{ }^{1} \mathrm{H}-\mathrm{NMR}\left(400 \mathrm{MHz}, \mathrm{CDCl}_{3}\right) \delta=10.46$ (s, imidazole), 7.37 ( $\mathrm{m}_{\mathrm{c}}, 2 \mathrm{H}$, imidazole), 5.23 (dd t, H-3), 4.90 (dd, H-2), 4.70 (dd, H-6A), 4.70 (dd $\sim$, H-4), 4.63 4.45 (m, 3 H, H-6B, $\mathrm{CH}_{2} \mathrm{~N}_{\text {imidazole }}$ ), 4.56 (d, H-1), 4.05 (ddd, H-5), 3.96 ( $\mathrm{m}_{\mathrm{c}}, 2 \mathrm{H}, \mathrm{CH}_{2} \mathrm{O}$ ), 3.77-3.69 (m, $\left.3 \mathrm{H}, \mathrm{EG}-\mathrm{CH}_{2}, \alpha-\mathrm{CH}_{2}-\mathrm{A}\right), 3.40\left(\mathrm{t}, 2 \mathrm{H}, \mathrm{CH}_{2} \mathrm{~N}_{3}\right), 3.33\left(\mathrm{dd}, \alpha-\mathrm{CH}_{2}-\mathrm{B}\right), 2.27,2.01,1.97(3 \mathrm{~s}, 3 \times 3 \mathrm{H}$, Ac), $1.52\left(\mathrm{~m}_{\mathrm{c}}, \beta-\mathrm{CH}\right), 1.24\left(\mathrm{~m}_{\mathrm{c}}, 24 \mathrm{H}\right.$, bulk- $\left.\mathrm{CH}_{2}\right), 0.87\left(\mathrm{t}, 6 \mathrm{H}, \mathrm{CH}_{3}\right) ;{ }^{3} J_{1,2}=8.0,{ }^{3} J_{2,3}=9.5$, ${ }^{3} J_{3,4}=9.5,{ }^{3} J_{4,5}=10.0,{ }^{3} J_{5,6 \mathrm{~A}}=4.0,{ }^{3} J_{5,6 \mathrm{~B}}=4.5,{ }^{2} J_{6}=14.0,{ }^{3} J_{\alpha \mathrm{B}, \beta}=6.0,{ }^{2} J_{\alpha}=9.5 \mathrm{~Hz} .{ }^{13} \mathrm{C} \mathrm{NMR}$ $\left(100 \mathrm{MHz}, \mathrm{CDCl}_{3}\right) \delta=170.6,169.8,169.2(\mathrm{CO}), 138.5$ (imidazole-CHN $\left.{ }_{2}\right), 122.9,122.6$ (imidazole), $101.1(\mathrm{C}-1), 73.5 / 73.4(\alpha), 72.2$ (C-3), $71.4(\mathrm{C}-5), 71.0(\mathrm{C}-2), 70.2\left(\mathrm{EG}^{\left.-\mathrm{CH}_{2}\right),} 68.9\left(\mathrm{CH}_{2} \mathrm{O}\right), 68.4\right.$ (C-4), $50.5\left(\mathrm{CH}_{2} \mathrm{~N}_{3}\right), 50.2\left(\mathrm{CH}_{2} \mathrm{~N}_{\text {imidazole }}\right), 49.6$ (C-6), 38.0 ( $\left.\beta\right), 31.9(\omega-2), 31.0,30.8,30.02$ / 30.00, 29.67 / 29.65, 29.59, 29.3 (bulk- $\mathrm{CH}_{2}$ ), 26.84 / 26.77 / 26.68 / 26.63 ( $\gamma$ ), $22.6(\omega-1), 21.4,20.52,20.48$ (Ac), $14.1(\omega)$.

11b $\mathbf{b}_{2}:[\propto]_{\mathrm{D}}^{25}=-13\left(\mathrm{c} \mathrm{0.12}, \mathrm{CH}_{3} \mathrm{OH}\right)$. IR [ATR, neat] $v / \mathrm{cm}^{-1} 3365(\mathrm{OH}), 2955,2924,2855(\mathrm{CH}), 2108$ $\left(\mathrm{N}_{3}\right) .{ }^{1} \mathrm{H}-\mathrm{NMR}\left(400 \mathrm{MHz}, \mathrm{CD}_{3} \mathrm{OD}\right) \delta=8.55$ (bs, <1 H, imidazole), 7.70, 7.60 (2 d, $2 \mathrm{H}$, imidazole), 4.63 (dd bd, H-6A), 4.46 (t, $2 \mathrm{H}, \mathrm{CH}_{2} \mathrm{~N}_{\text {imidazole }}$ ), 4.41 (dd, H-6B), 4.24 (d, H-1), 3.89 (t, $2 \mathrm{H}, \mathrm{CH}_{2} \mathrm{O}$ ), 3.74-3.64 (m, 3 H, $\mathrm{CH}_{2} \mathrm{~N}_{3}, \alpha-\mathrm{CH}_{2}-\mathrm{A}$ ), 3.61 (ddd, H-5), 3.38 (dd $\sim \mathrm{t}, \mathrm{H}-3$ ), 3.37 (dd, $\beta-\mathrm{CH}_{2}-\mathrm{B}$ ), 3.15 (dd, H-2), 3.06 (dd t, H-4), $1.59\left(\mathrm{~m}_{\mathrm{c}}, \beta-\mathrm{CH}\right), 1.30\left(\mathrm{~m}_{\mathrm{c}}, 24 \mathrm{H}\right.$, bulk-CH$), 0.90\left(\mathrm{t}, 6 \mathrm{H}, \mathrm{CH}_{3}\right)$; ${ }^{3} J_{1,2}=8.0,{ }^{3} J_{2,3}=9.0,{ }^{3} J_{3,4}=9.0,{ }^{3} J_{4,5}=9.5,{ }^{3} J_{5,6 \mathrm{~A}}=2.5,{ }^{3} J_{5,6 \mathrm{~B}}=7.0,{ }^{2} J_{6}=14.5,{ }^{3} J_{\alpha, \beta}=2.0,{ }^{2} J_{\alpha}=14.5$, ${ }^{3} J_{\mathrm{CH} 2 \mathrm{~N}, \mathrm{CH} 2 \mathrm{O}}=4.5 \mathrm{~Hz} .{ }^{13} \mathrm{C} \mathrm{NMR}\left(100 \mathrm{MHz}, \mathrm{CD}_{3} \mathrm{OD}\right) \delta=125.0,123.9$ (imidazole), $105.0(\mathrm{C}-1), 77.8$ (C-3), $75.1(\mathrm{C}-2), 74.9(\mathrm{C}-5), 74.3(\alpha), 72.5(\mathrm{C}-4), 71.4\left(\mathrm{EG}-\mathrm{CH}_{2}\right), 69.8\left(\mathrm{CH}_{2} \mathrm{O}\right), 51.9\left(\mathrm{CH}_{2} \mathrm{~N}_{3}\right), 51.8$ (C-6), $51.1\left(\mathrm{CH}_{2} \mathrm{~N}_{\text {imidazole }}\right), 39.7$ ( $\beta$ ), 33.2 ( $\left.\omega-2\right), 32.36$ / 32.34, 32.30 / 32.28, 31.3, 31.0, 30.88 /

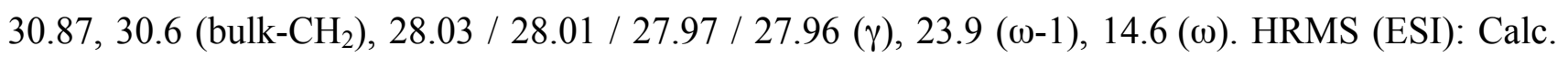
for $[\mathrm{M}-\mathrm{Br}]\left[\mathrm{C}_{29} \mathrm{H}_{54} \mathrm{~N}_{5} \mathrm{O}_{6}\right]^{+}$568.4074; found 568.4056. 
Micelle conjugation and reference compound

2-Butyl-octyl 6-\{1-[11-(4-hydroxymethyl-1H-1,2,3-triazole-1-yl)-3,6,9-trioxa-undecyl]imidazolium-3-yl\}-6-deoxy- $\beta$-D-glucopyranoside bromide (14). ${ }^{1} \mathrm{H}$ NMR (400 $\left.\mathrm{MHz}, \mathrm{CD}_{3} \mathrm{OD}\right): \delta$ 8.95 (s, imidazole- $\mathrm{CHN}_{2}$ ), 7.97 (s, triazole), 7.68, 7.56 (2s, $2 \mathrm{H}$, imidazole), $4.67\left(\mathrm{~m}_{\mathrm{c}}, 3 \mathrm{H}, \mathrm{CH}_{2} \mathrm{OH}\right.$, H-6A), 4.59 (t, $2 \mathrm{H}, \mathrm{CH}_{2} \mathrm{~N}_{\text {trizole }}$ ), 4.40 ( $\left.\mathrm{m}_{\mathrm{c}}, 3 \mathrm{H}, \mathrm{CH}_{2} \mathrm{~N}_{\text {imidazole }}, \mathrm{H}-6 \mathrm{~B}\right), 4.25$ (d, H-1), 3.93-3.90 (m, >2 $\mathrm{H}, \mathrm{CH}_{2} \mathrm{O}$ ), 3.66-3.53 (m, >12 H, EG-CH $2, \mathrm{H}-5, \alpha-\mathrm{CH}_{2}-\mathrm{A}$ ), 3.40-3.35 (m, $\left.2 \mathrm{H}, \mathrm{H}-3, \alpha-\mathrm{CH}_{2}-\mathrm{B}\right), 3.13$ (dd, H-2), 3.04 (dd t, H-4), 1.55-1.48 (m, $\beta-\mathrm{CH}), 1.29\left(\mathrm{~m}_{\mathrm{c}},>14 \mathrm{H}\right.$, bulk- $\left.\mathrm{CH}_{2}\right), 0.93\left(\mathrm{~m}_{\mathrm{c}}, 6 \mathrm{H}, \mathrm{CH}_{3}\right)$. HRMS (ESI): Calc. for [M-Br] $\left[\mathrm{C}_{32} \mathrm{H}_{58} \mathrm{~N}_{5} \mathrm{O}_{9}\right]^{+}$656.4231, 657.4269 (35\%); found 565.4231, $657.4257(32 \%)$.

1,8-Diazido-3,6-dioxa-octane (15). A solution of 1,2-bis-(2-chloroethoxy)-ethane (3.0 mL, $19 \mathrm{mmol})$ in DMF $(50 \mathrm{~mL})$ was treated with $\mathrm{NaN}_{3}(3.7 \mathrm{~g}, 57 \mathrm{mmol})$ and the reaction was heated to $80{ }^{\circ} \mathrm{C}$ overnight. The solvent was evaporated at reduced pressure and the residue extracted with $\mathrm{CH}_{2} \mathrm{Cl}_{2}$ to furnish 15 as colourless liquid (3.7 g, 97\%). ${ }^{1} \mathrm{H}$ NMR (400 MHz, $\left.\mathrm{CDCl}_{3}\right)$ : $\delta 3.68-3.65(\mathrm{~m}$, $\left.8 \mathrm{H}, \mathrm{OCH}_{2}\right), 3.37\left(\mathrm{t}, 4 \mathrm{H}, \mathrm{CH}_{2} \mathrm{~N}_{3}\right) .{ }^{13} \mathrm{C} \mathrm{NMR}\left(100 \mathrm{MHz}, \mathrm{CDCl}_{3}\right): \delta 70.8,70.2\left(-\mathrm{OCH}_{2}\right), 50.8\left(\mathrm{CH}_{2} \mathrm{~N}_{3}\right)$.

1,8-Bis-(4-hydroxymethyl-1,2,3-triazole-1-yl)-3,6-dioxa-octane (16). A solution of $\mathbf{1 5}$ (0.50 g, 2.5 mmol) and propargyl alcohol $(0.36 \mathrm{~mL}, 6.2 \mathrm{mmol})$ in $\mathrm{CH}_{3} \mathrm{OH}(20 \mathrm{~mL})$ was treated with $\mathrm{Cu}(\mathrm{OAc})_{2}$ (70 $\mathrm{mg}, 0.4 \mathrm{mmol})$ and sodium ascorbate $(0.22 \mathrm{~g}, 1.1 \mathrm{mmol})$ under ice-bath cooling. After $30 \mathrm{~min}$ the reaction was alowed to warm to $\mathrm{rt}$ and stirred overnight. Methanol was evaporated at reduced pressure and the residue was distributed between butanol and water. The organic layer was dried over $\mathrm{MgSO}_{4}$ and concentrated to furnish crude triazole 16 as yellow liquid (0.58 $\left.\mathrm{g}, 74 \%\right)$. NMR-analysis revealed that the product contained minor contents of the solvent $\left({ }^{\mathrm{n}} \mathrm{BuOH}\right) .{ }^{1} \mathrm{H}$ NMR (400 MHz, $\mathrm{CD}_{3} \mathrm{OD}$ ): $\delta 7.93$ (s, $2 \mathrm{H}$, triazole), $4.68\left(\mathrm{~s}, 4 \mathrm{H}, \mathrm{CH}_{2} \mathrm{OH}\right), 4.55\left(\mathrm{t}, 4 \mathrm{H}, \mathrm{CH}_{2} \mathrm{~N}_{\text {triazole }}\right.$ ), 3.83 (t, $4 \mathrm{H}, \mathrm{CH}_{2} \mathrm{O}$ ), 3.56 (m, $\left.4 \mathrm{H}, \mathrm{EG}-\mathrm{CH}_{2}\right) .{ }^{13} \mathrm{C}$ NMR (100 MHz, $\mathrm{CD}_{3} \mathrm{OD}$ ): 149.1 (triazole-C), 124.9 (triazole-CH), $71.4\left(\mathrm{EG}-\mathrm{CH}_{2}\right), 70.5\left(\mathrm{CH}_{2} \mathrm{O}\right), 56.6\left(\mathrm{CH}_{2} \mathrm{OH}\right), 51.5\left(\mathrm{CH}_{2} \mathrm{~N}\right) . \mathrm{HRMS}$ (ESI): Calc. for $[\mathrm{M}+\mathrm{Na}]\left[\mathrm{C}_{12} \mathrm{H}_{20} \mathrm{~N}_{6} \mathrm{O}_{4} \mathrm{Na}\right]^{+}$335.1431, 336.1478 (13\%); found 335.1431, 336.1457 (14\%). 
NMR Spectra:
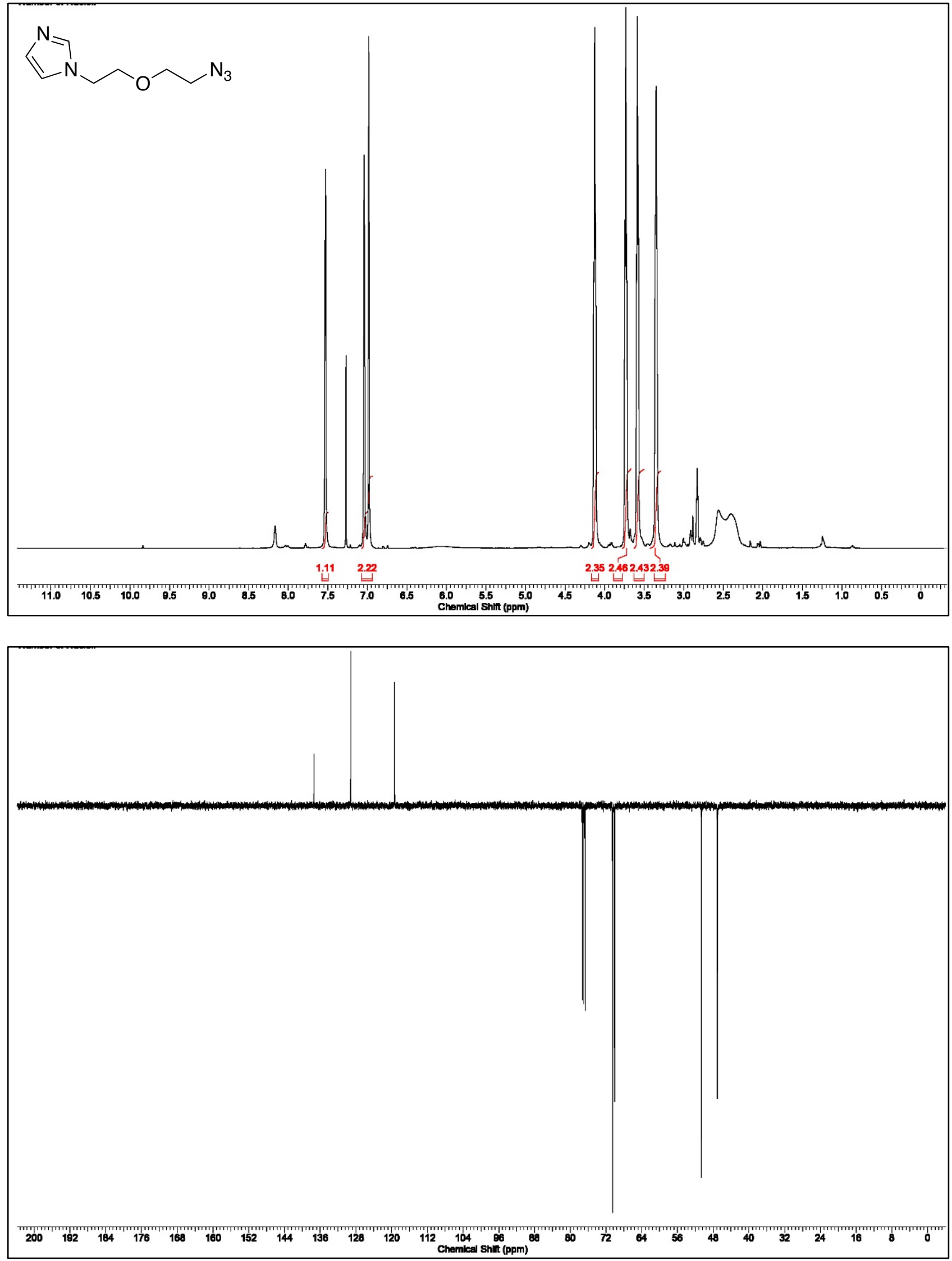

Figure S5. ${ }^{1} \mathrm{H} \& \mathrm{APT}-{ }^{13} \mathrm{C}$ NMR spectra of $\mathbf{9}_{2}$ 

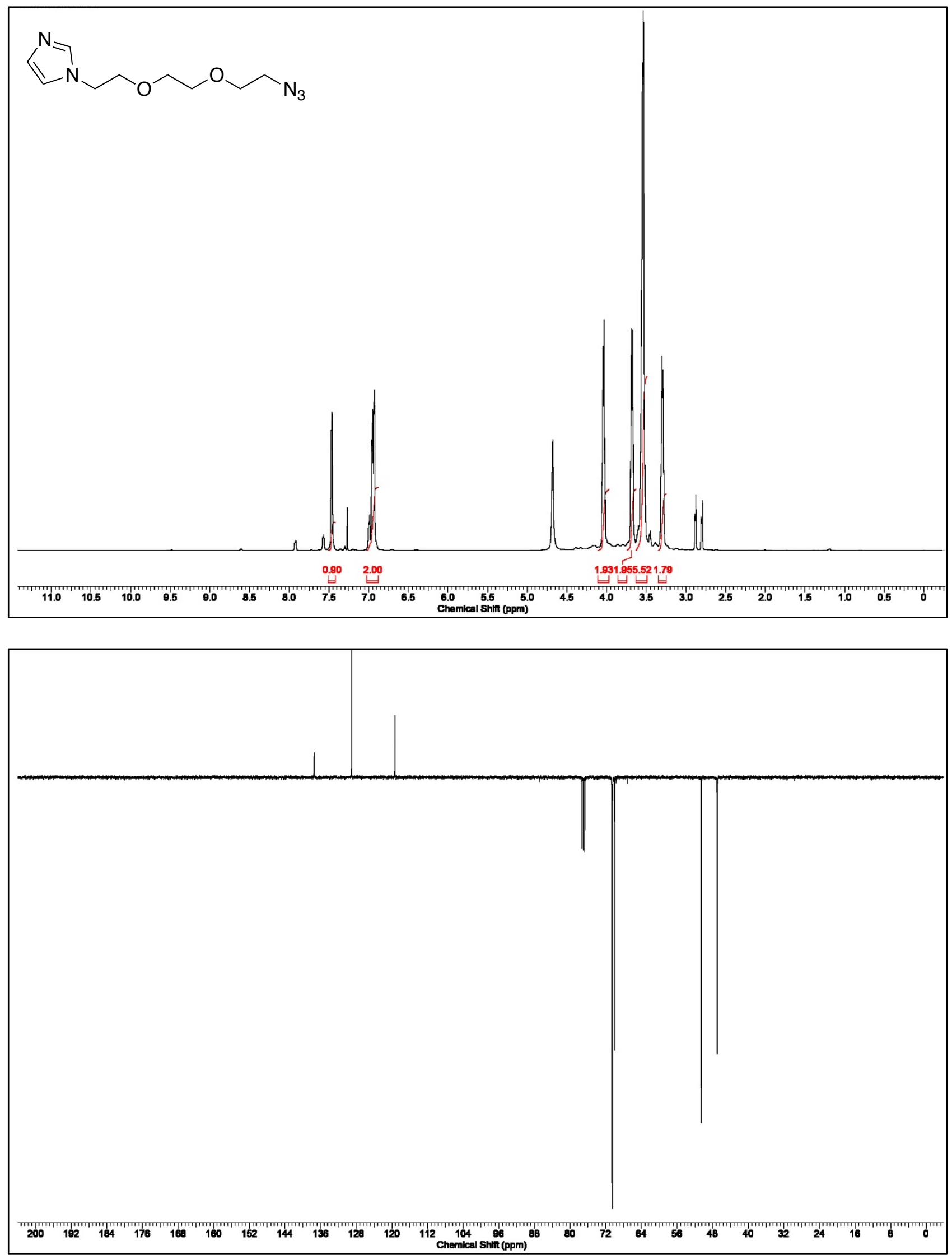

Figure S6. ${ }^{1} \mathrm{H} \& \mathrm{APT}-{ }^{13} \mathrm{C}$ NMR spectra of $\mathbf{9}_{3}$

- SB / 26 - 

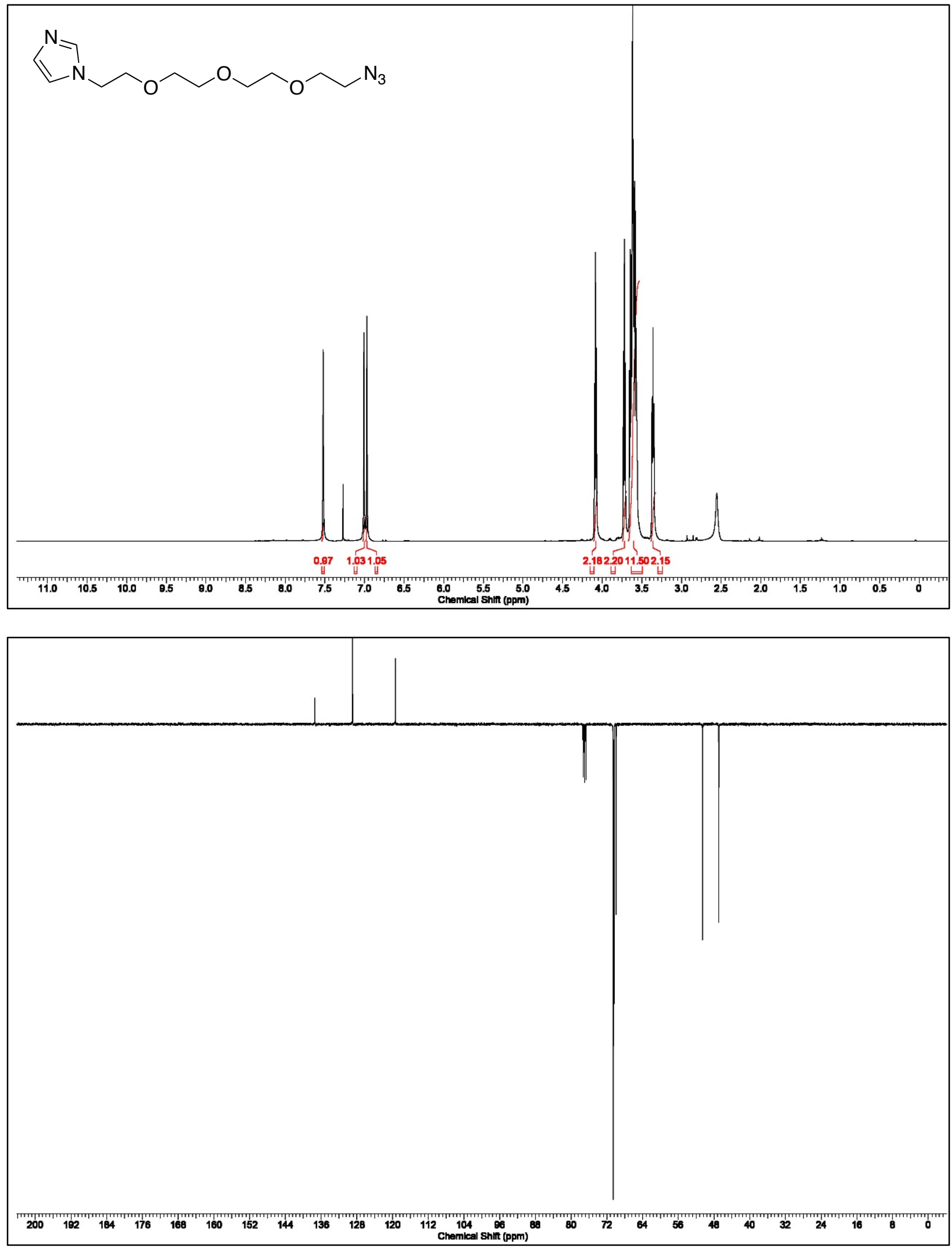

Figure S7. ${ }^{1} \mathrm{H} \& \mathrm{APT}-{ }^{13} \mathrm{C}$ NMR spectra of $\mathbf{9}_{4}$ 

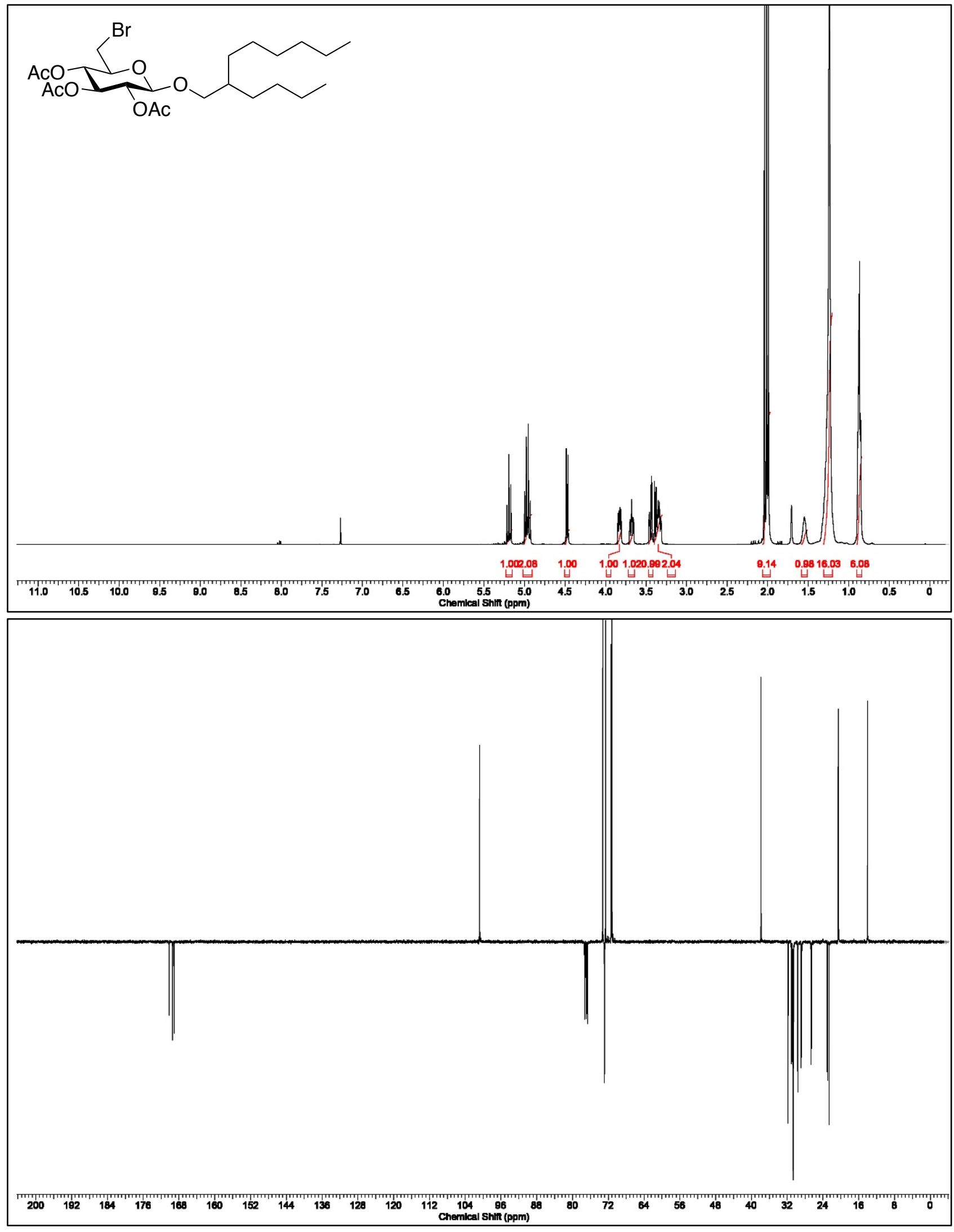

Figure S8. ${ }^{1} \mathrm{H} \& \mathrm{APT}-{ }^{13} \mathrm{C}$ NMR spectra of 5a 

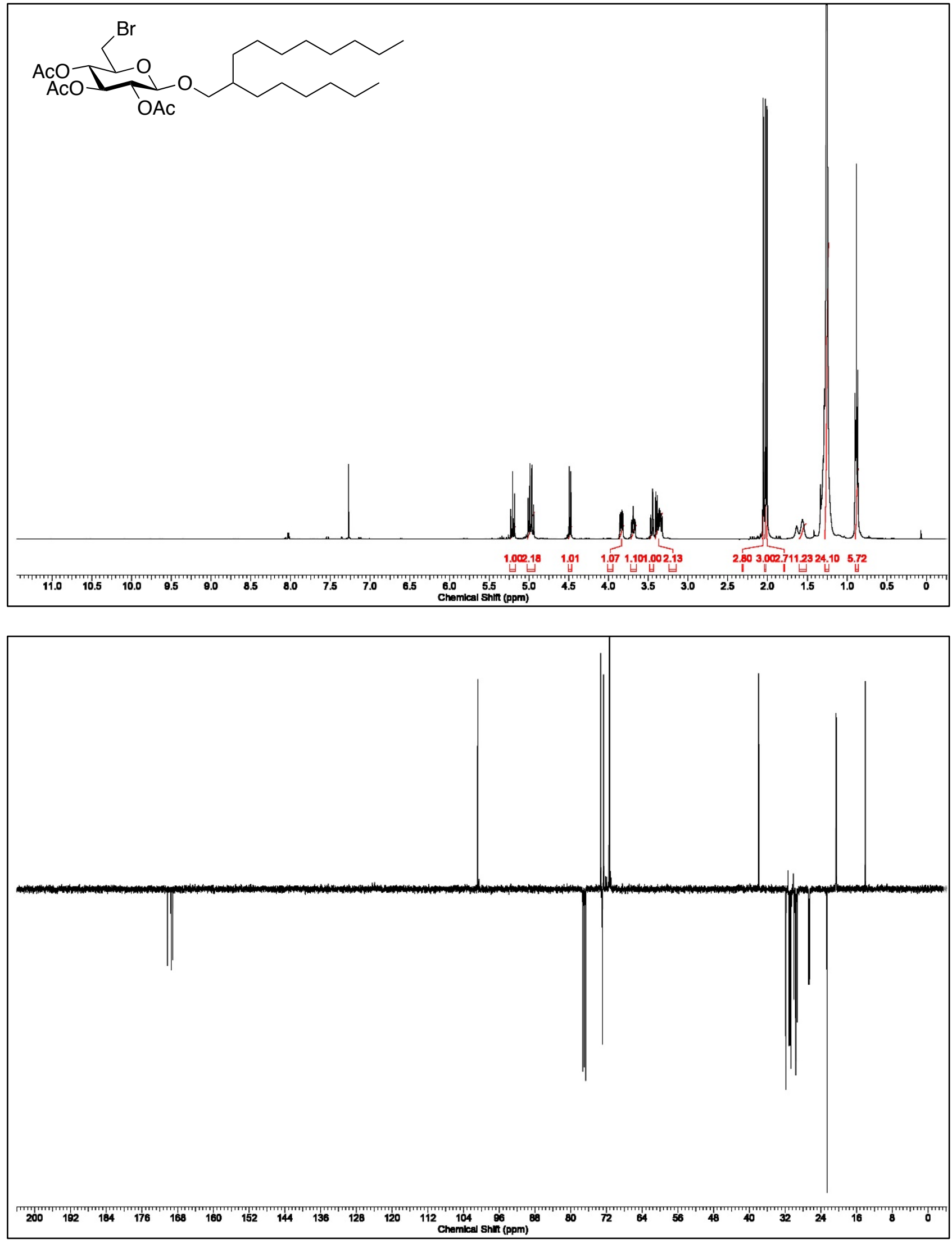

Figure 59. ${ }^{1} \mathrm{H} \& \mathrm{APT}-{ }^{13} \mathrm{C}$ NMR spectra of $\mathbf{5 b}$ 

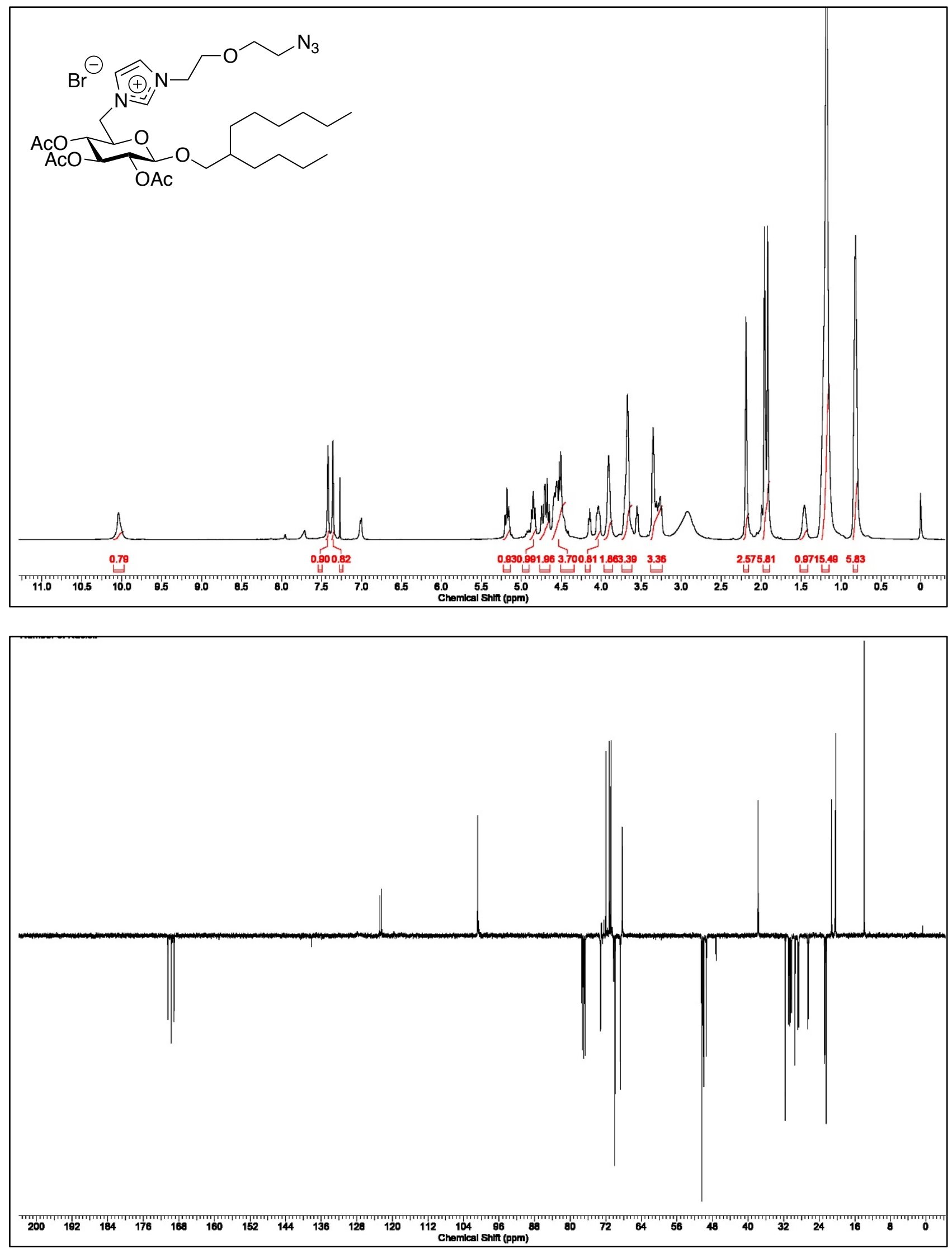

Figure S10. ${ }^{1} \mathrm{H} \& \mathrm{APT}-{ }^{13} \mathrm{C}$ NMR spectra of $\mathbf{1 0 a _ { 2 }}$ 

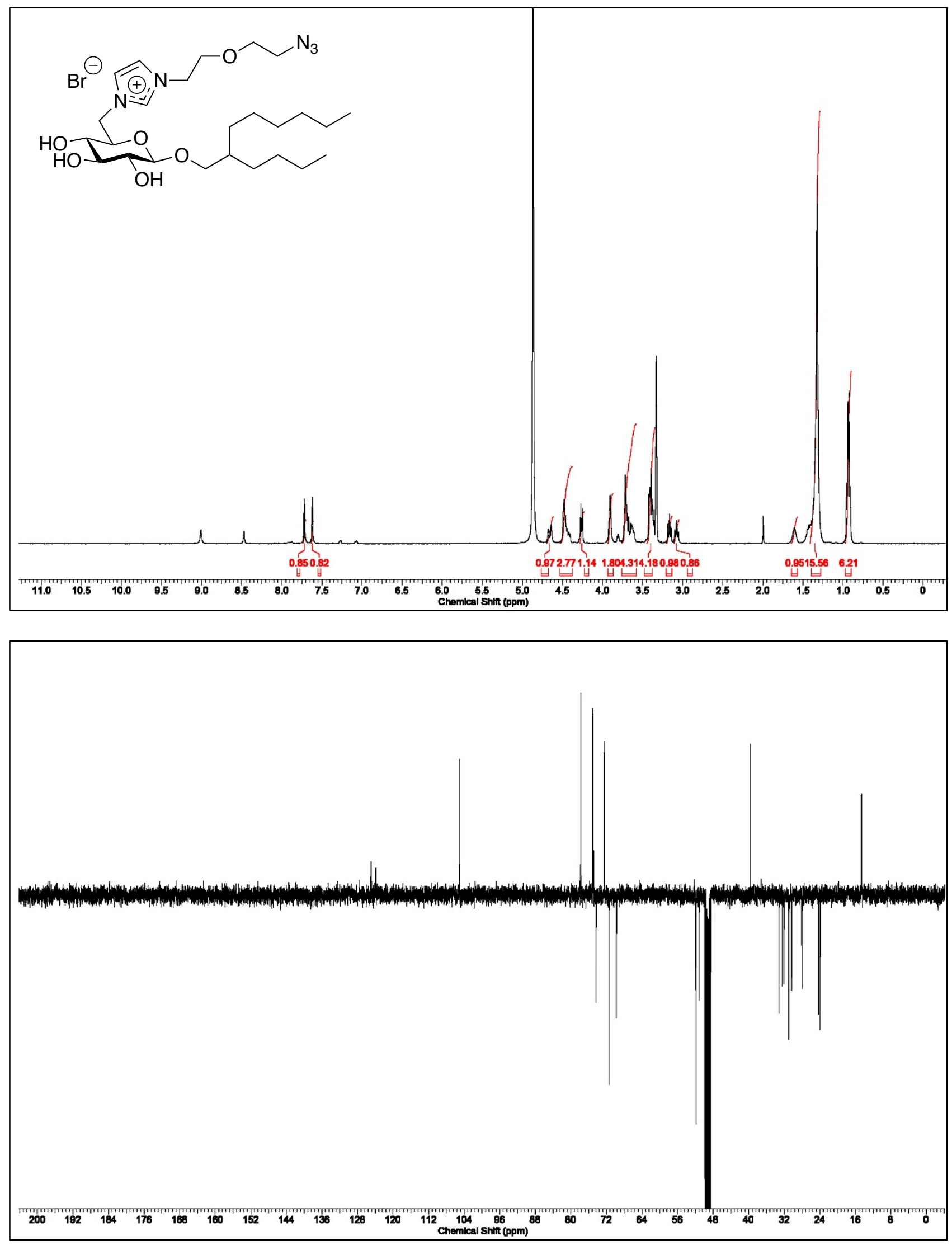

Figure S11. ${ }^{1} \mathrm{H} \& \mathrm{APT}-{ }^{13} \mathrm{C}$ NMR spectra of $\mathbf{1 1 a _ { 2 }}$ 

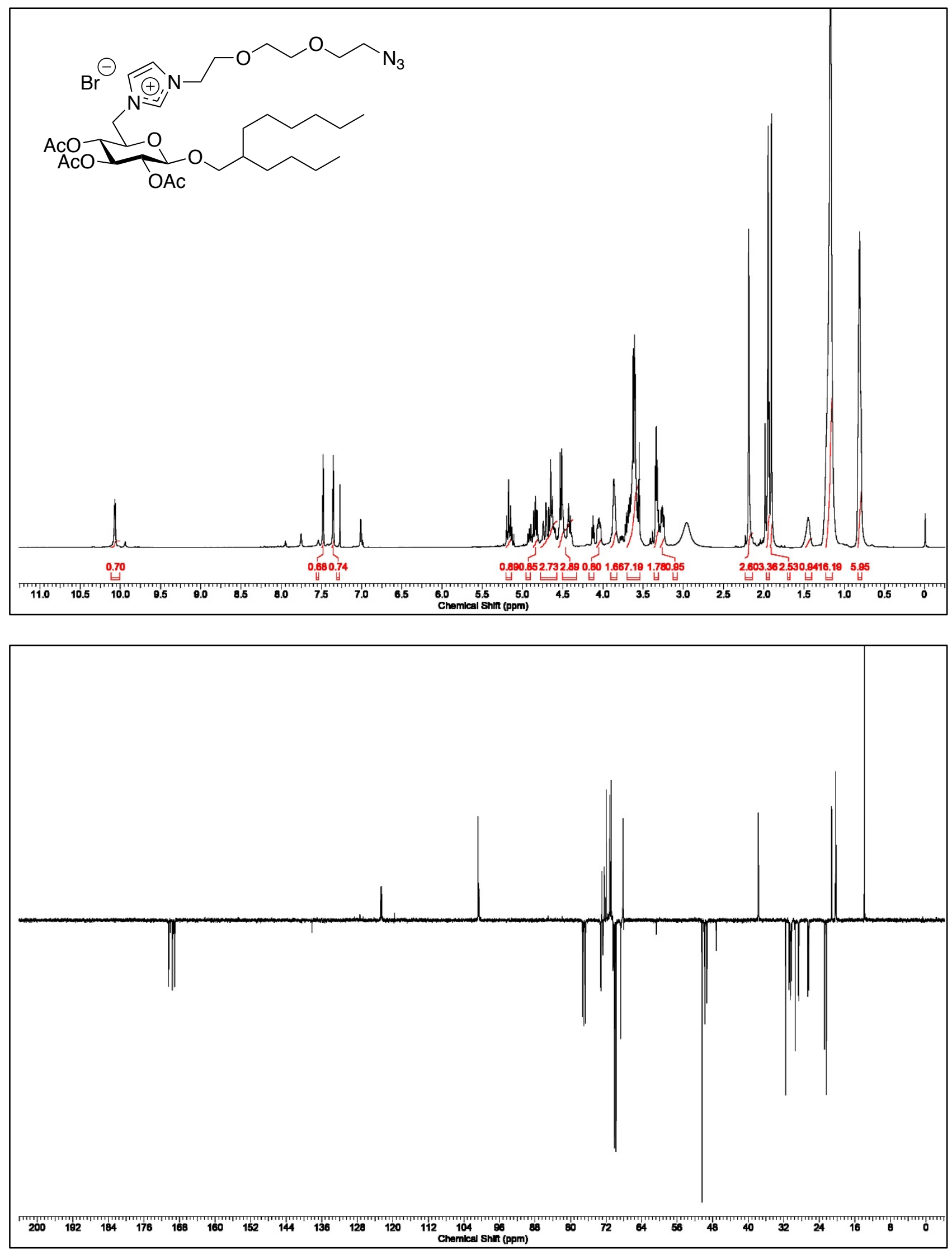

Figure S12. ${ }^{1} \mathrm{H} \& \mathrm{APT}-{ }^{13} \mathrm{C}$ NMR spectra of $\mathbf{1 0 a}_{3}$ 

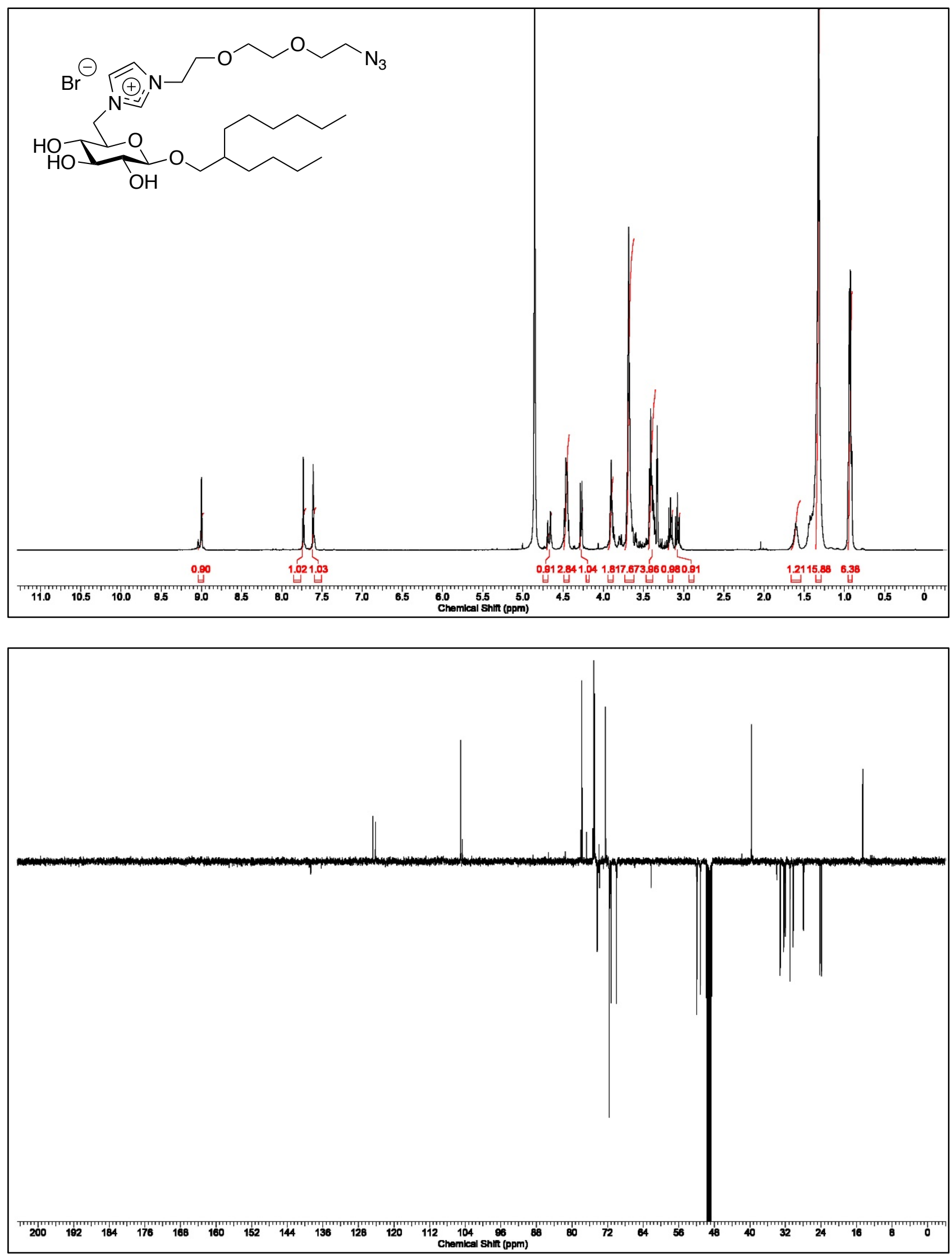

Figure S13. ${ }^{1} \mathrm{H} \& \mathrm{APT}-{ }^{13} \mathrm{C}$ NMR spectra of $\mathbf{1 1 a _ { 3 }}$ 

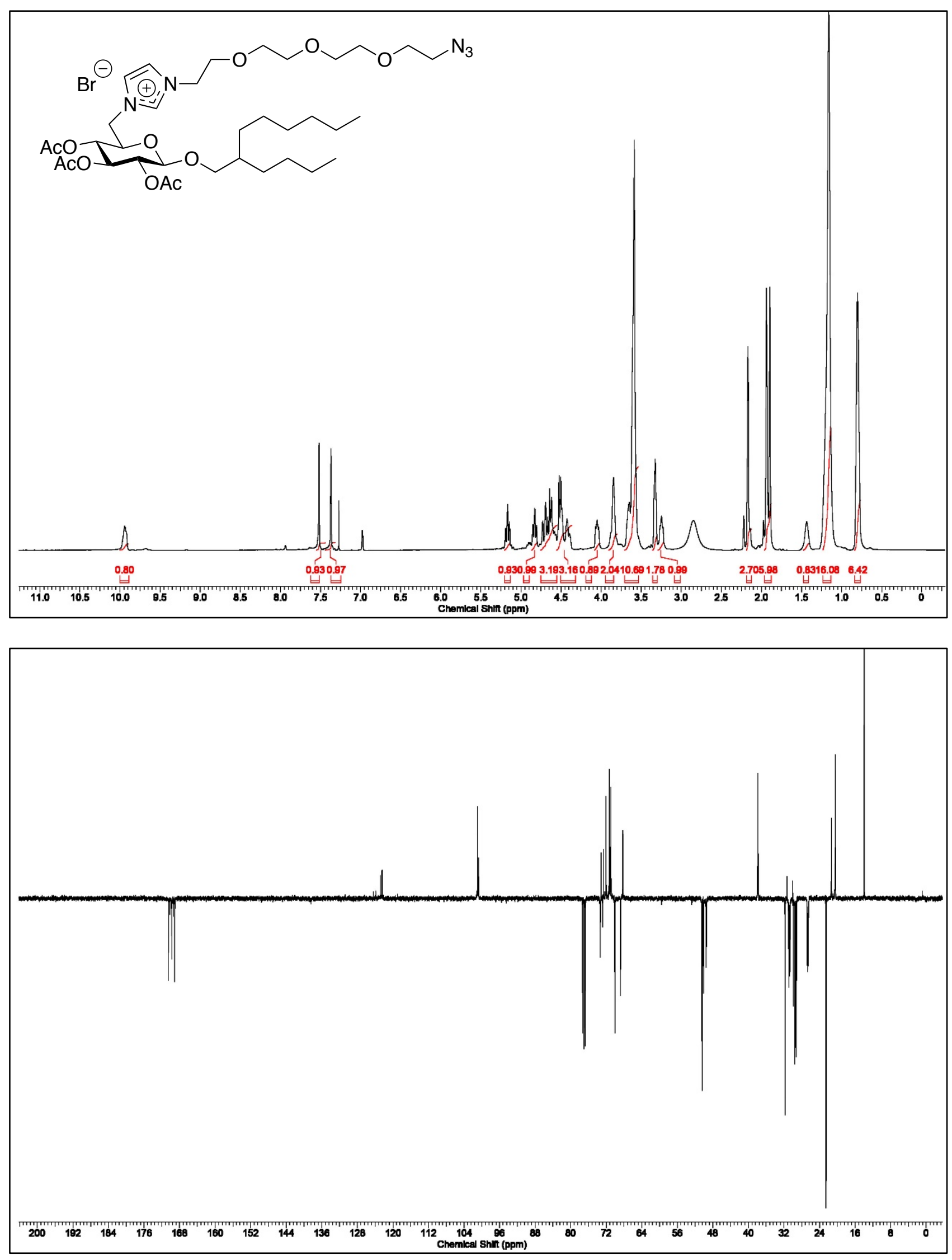

Figure S14. ${ }^{1} \mathrm{H} \& \mathrm{APT}-{ }^{13} \mathrm{C}$ NMR spectra of $\mathbf{1 0 a _ { 4 }}$ 

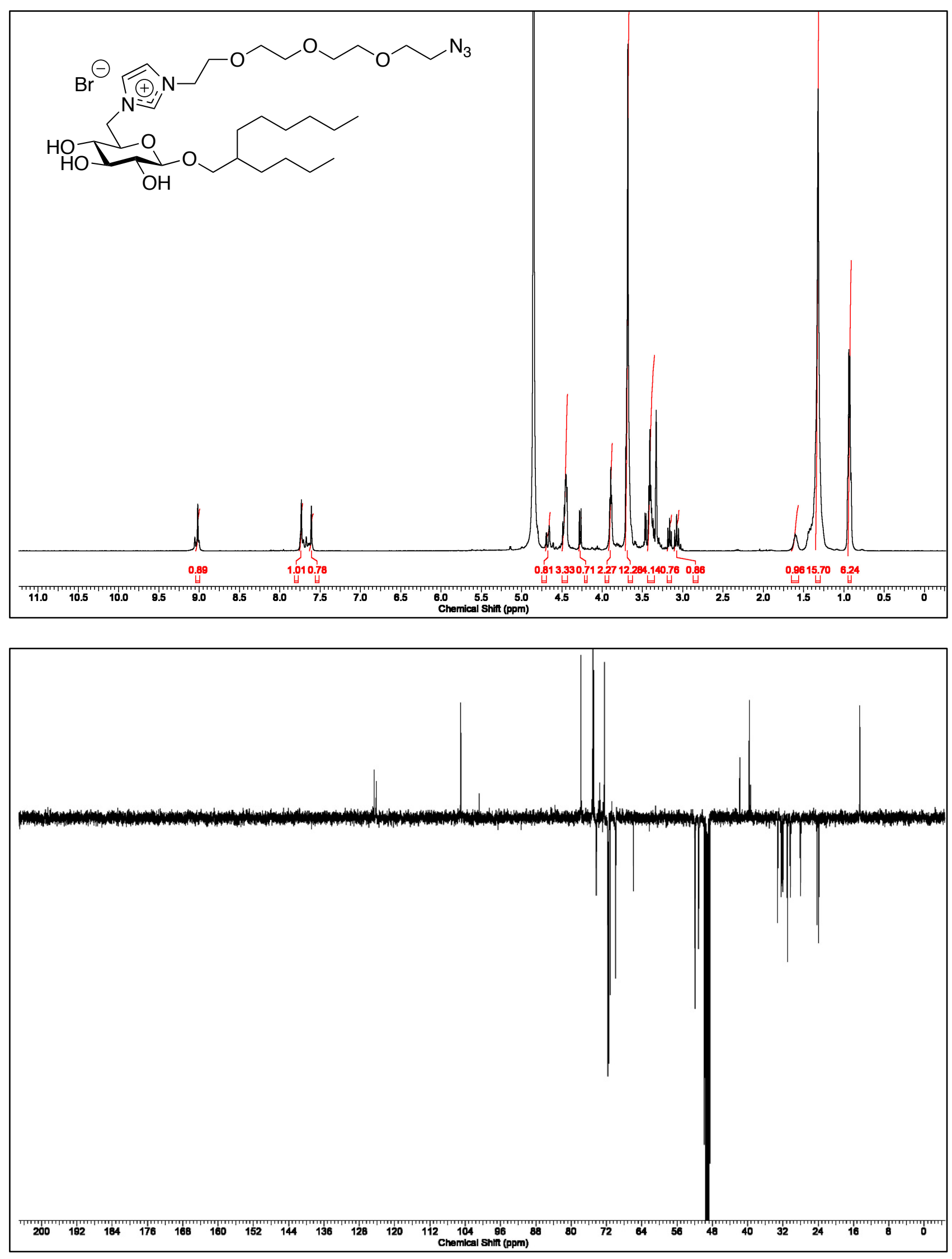

Figure S15. ${ }^{1} \mathrm{H} \& \mathrm{APT}-{ }^{13} \mathrm{C}$ NMR spectra of $\mathbf{1 1} \mathbf{a}_{4}$ 

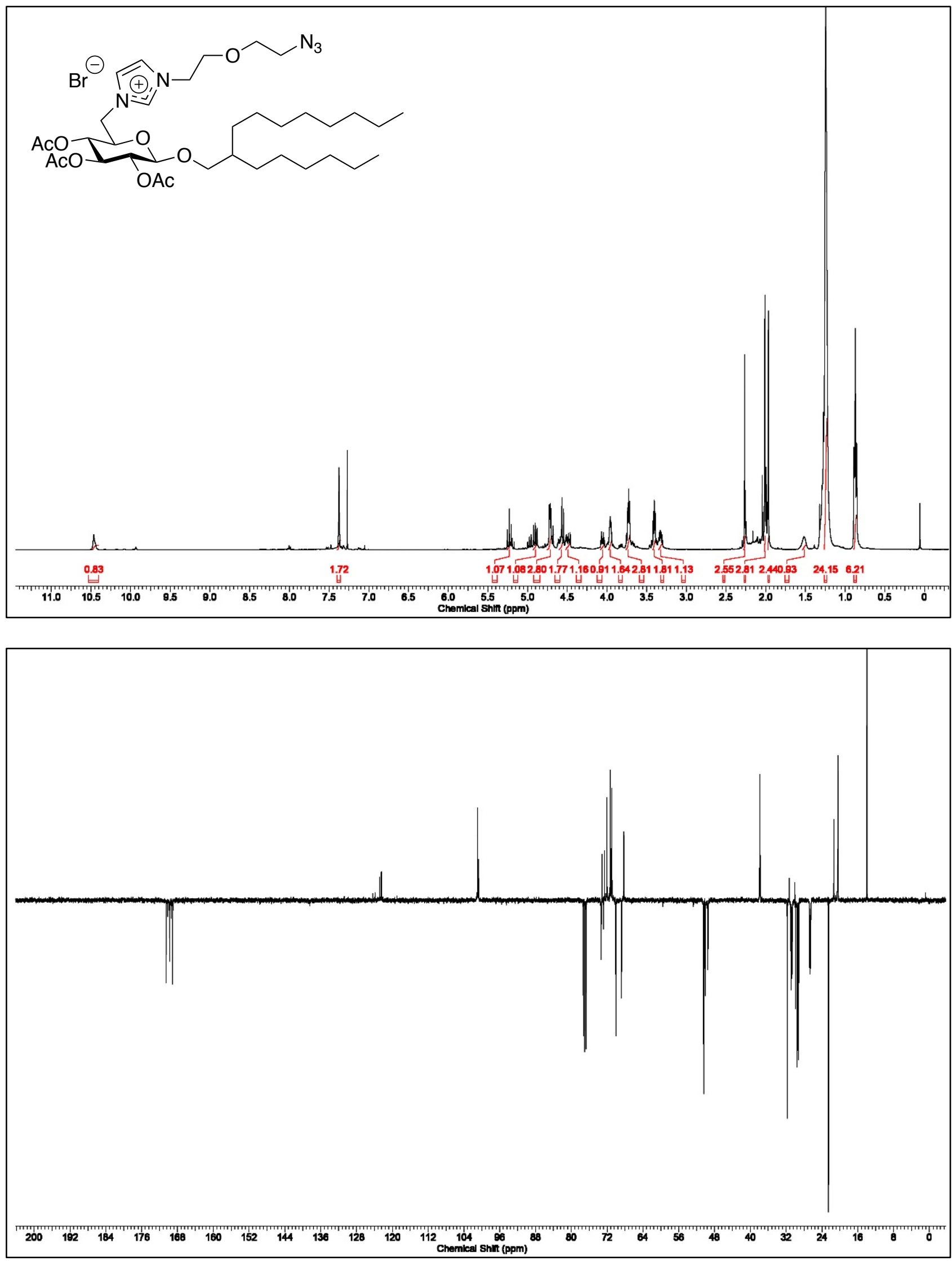

Figure S16. ${ }^{1} \mathrm{H} \& \mathrm{APT}-{ }^{13} \mathrm{C}$ NMR spectra of $\mathbf{1 0 b _ { 2 }}$ 

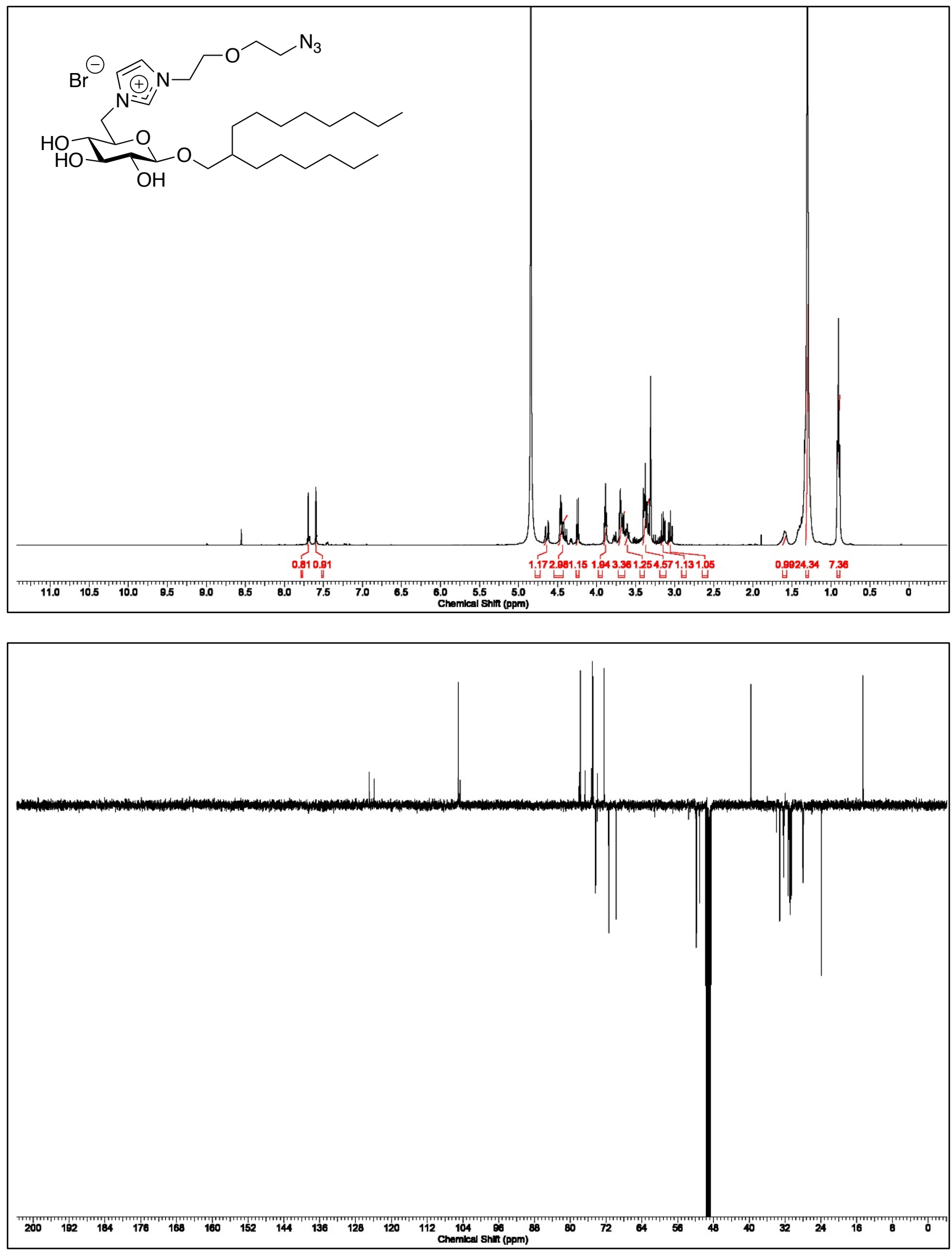

Figure S17. ${ }^{1} \mathrm{H} \& \mathrm{APT}-{ }^{13} \mathrm{C}$ NMR spectra of $\mathbf{1 1} \mathbf{b}_{2}$ 

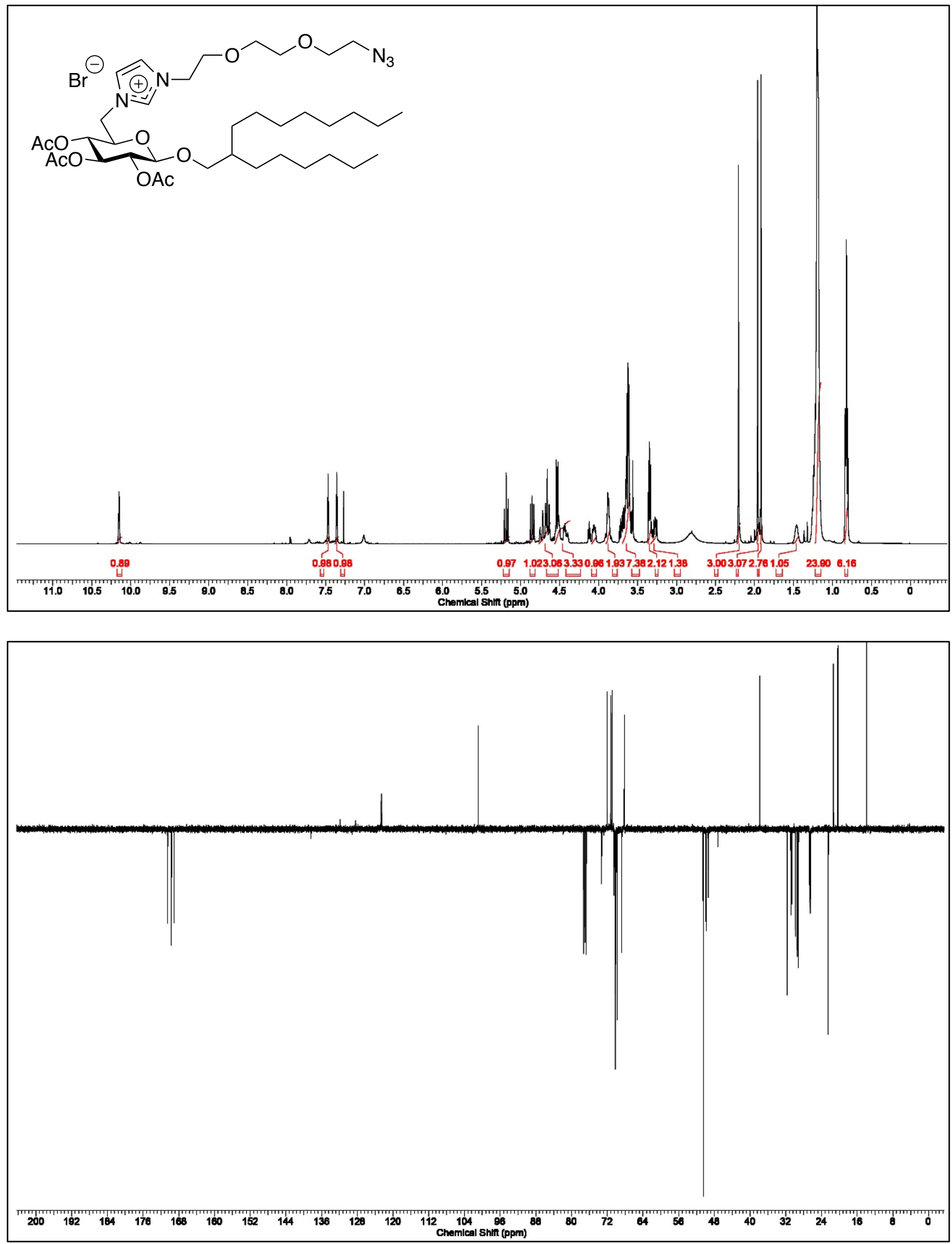

Figure S18. ${ }^{1} \mathrm{H} \& \mathrm{APT}-{ }^{13} \mathrm{C}$ NMR spectra of $\mathbf{1 0 b}_{\mathbf{3}}$ 

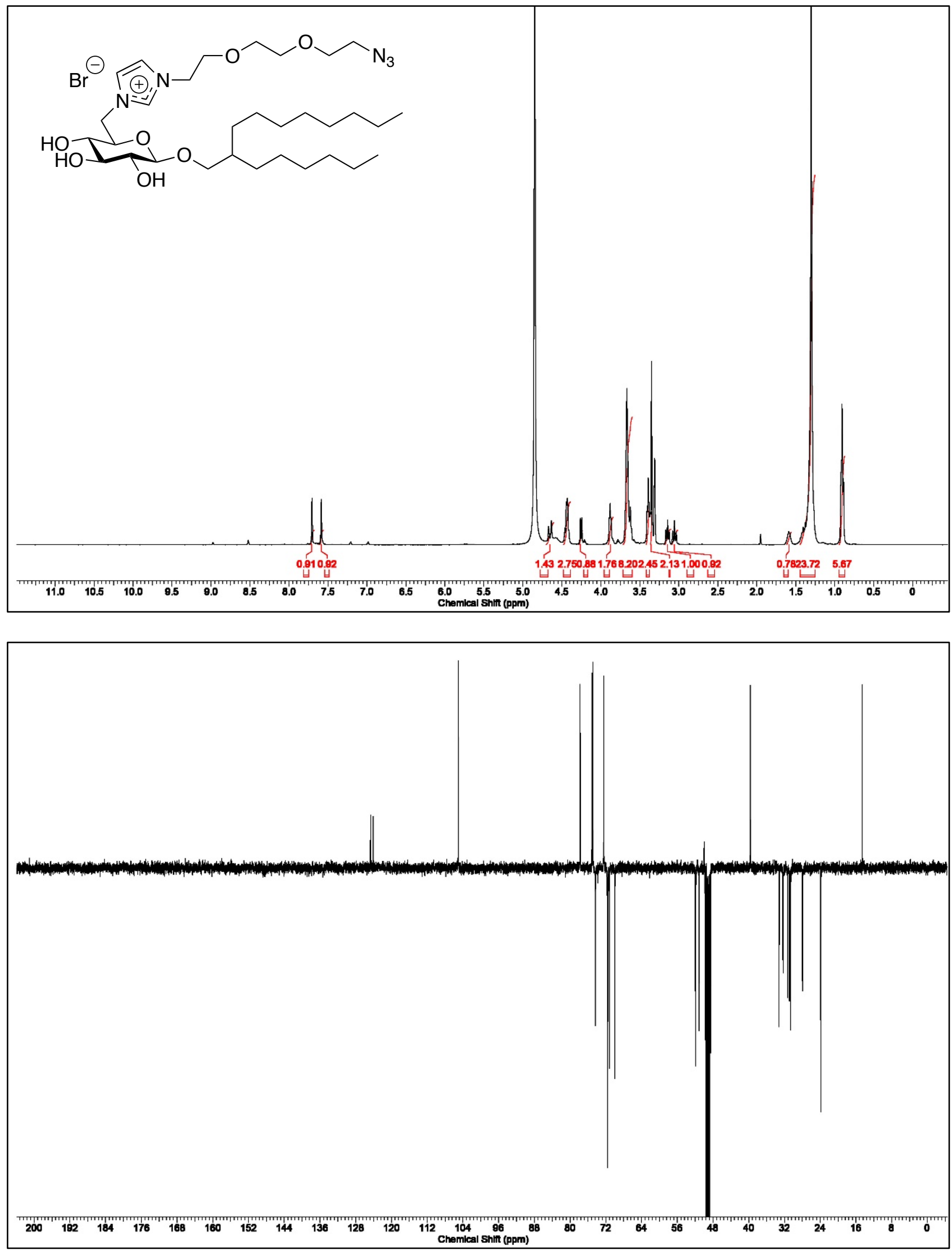

Figure S19. ${ }^{1} \mathrm{H} \& \mathrm{APT}-{ }^{13} \mathrm{C}$ NMR spectra of $\mathbf{1 1 b}_{\mathbf{3}}$ 

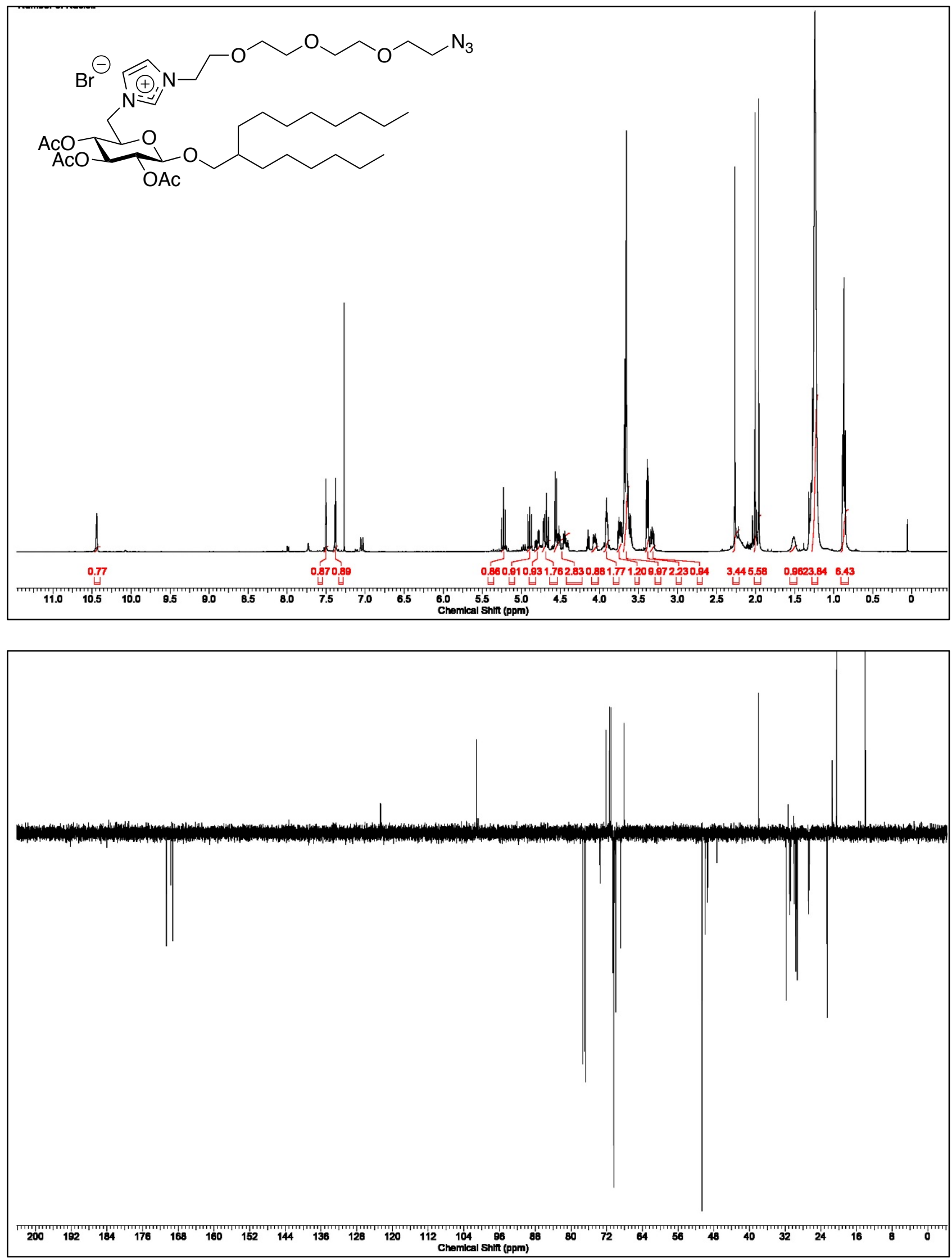

Figure S20. ${ }^{1} \mathrm{H} \& \mathrm{APT}-{ }^{13} \mathrm{C}$ NMR spectra of $\mathbf{1 0 b}_{4}$ 

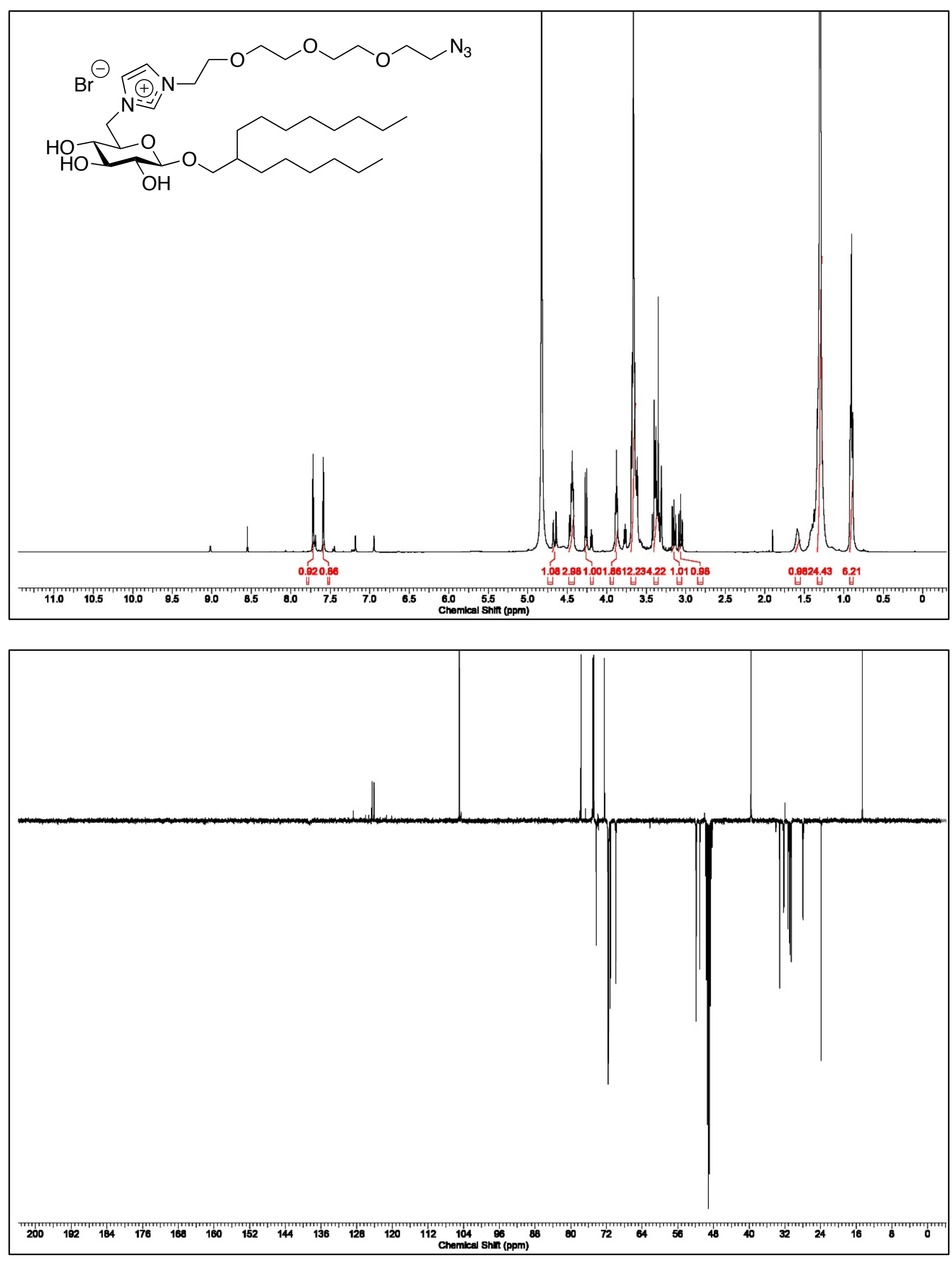

Figure S21. ${ }^{1} \mathrm{H} \& \mathrm{APT}-{ }^{13} \mathrm{C}$ NMR spectra of $\mathbf{1 1 b}_{4}$ 


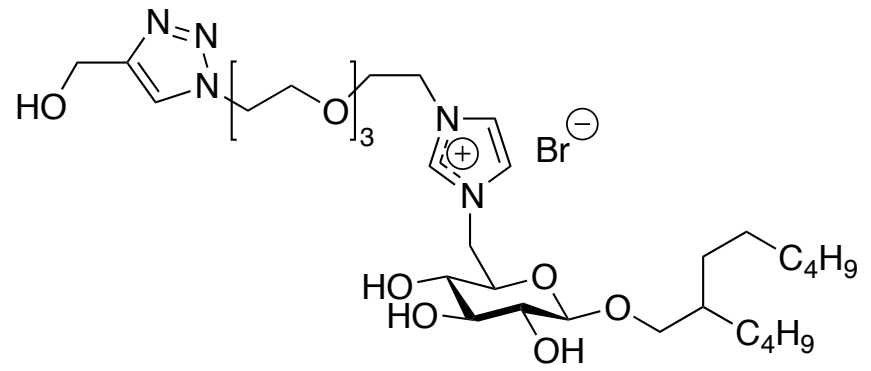

14
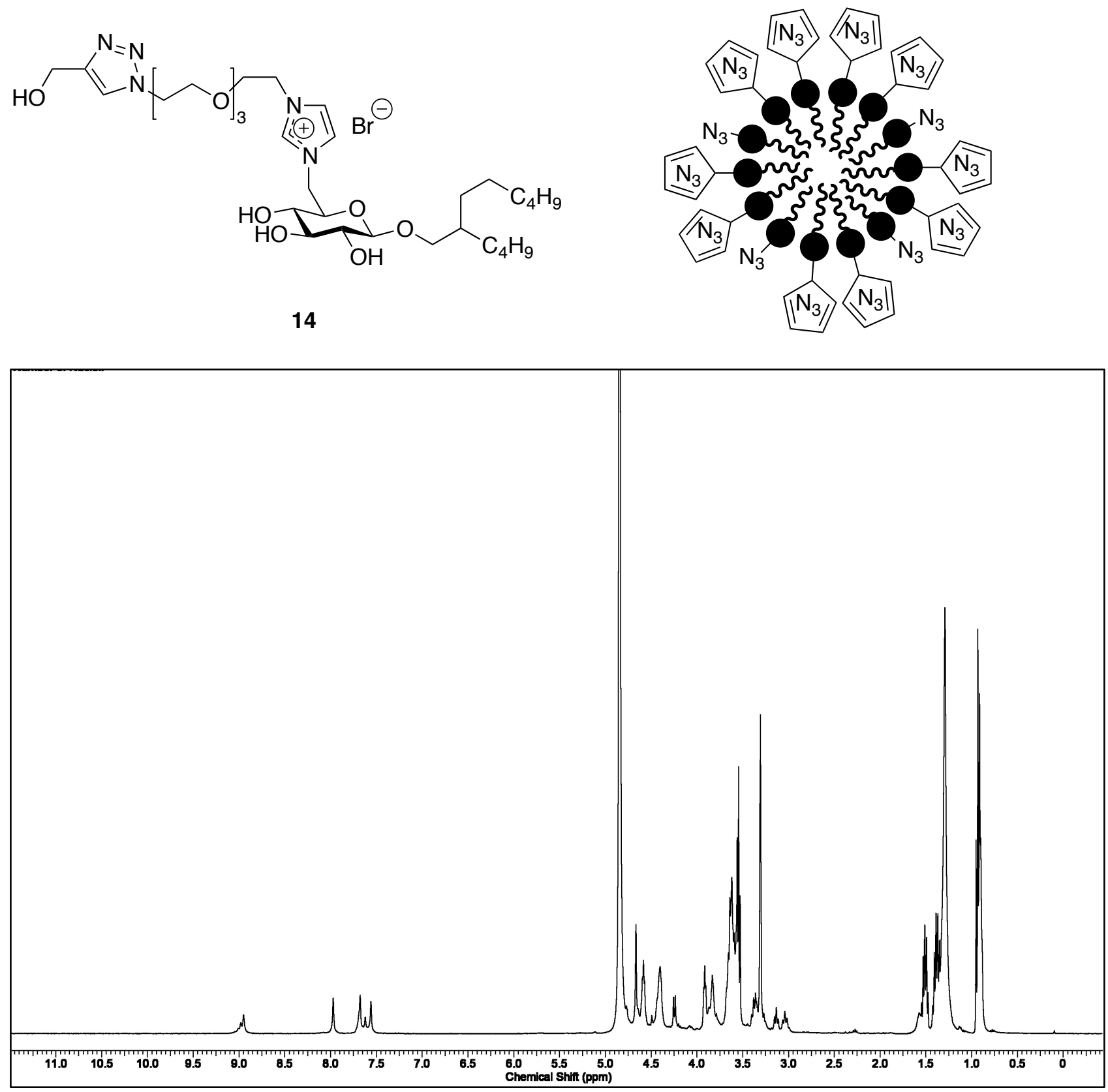

Figure 22. ${ }^{1} \mathrm{H}$ NMR spectra of $\mathbf{1 4}$ obtained by CLICK-coupling in micellar phase 

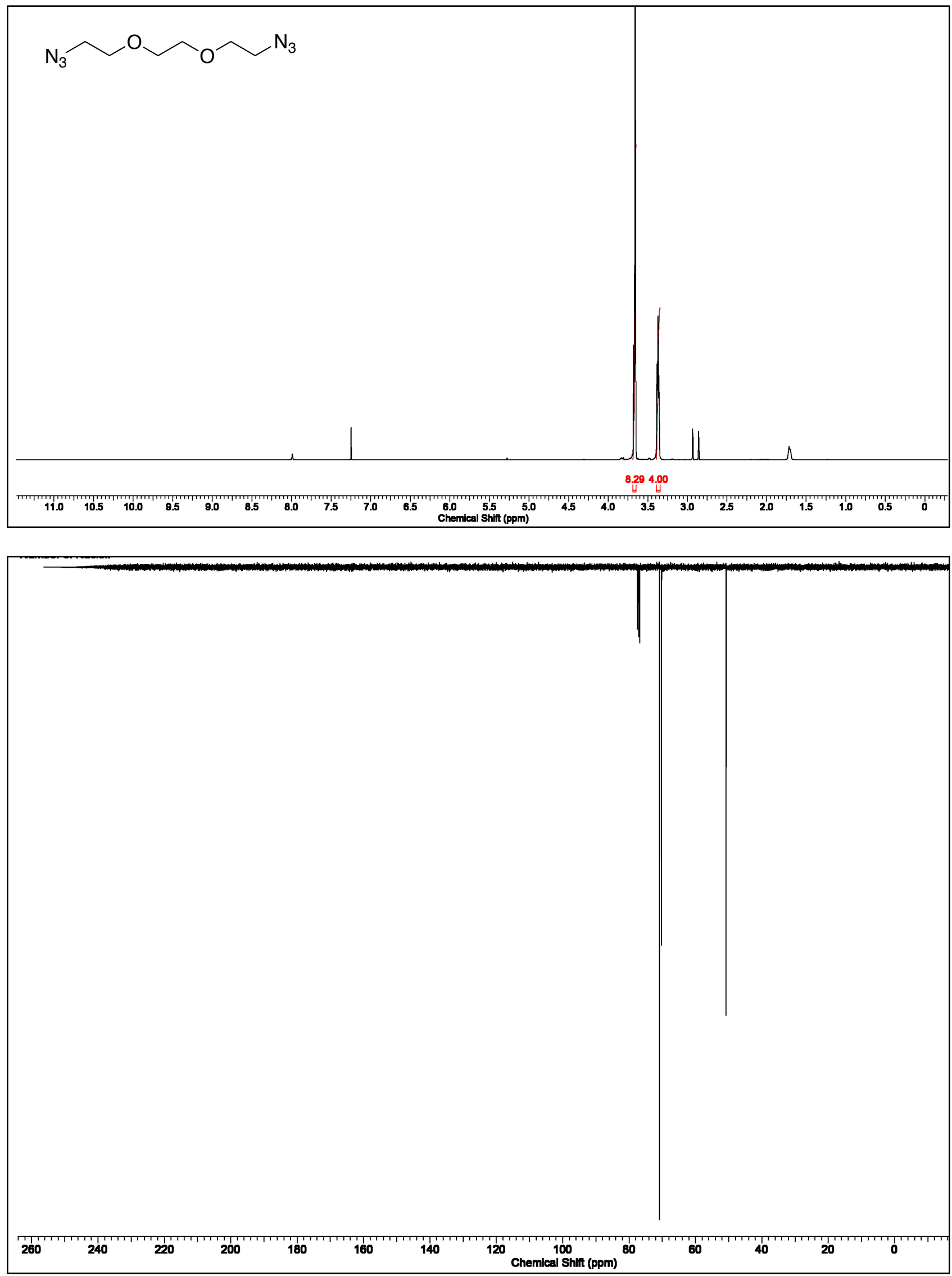

Figure S23. ${ }^{1} \mathrm{H} \& \mathrm{APT}-{ }^{13} \mathrm{C}$ NMR spectra of $\mathbf{1 5}$ 

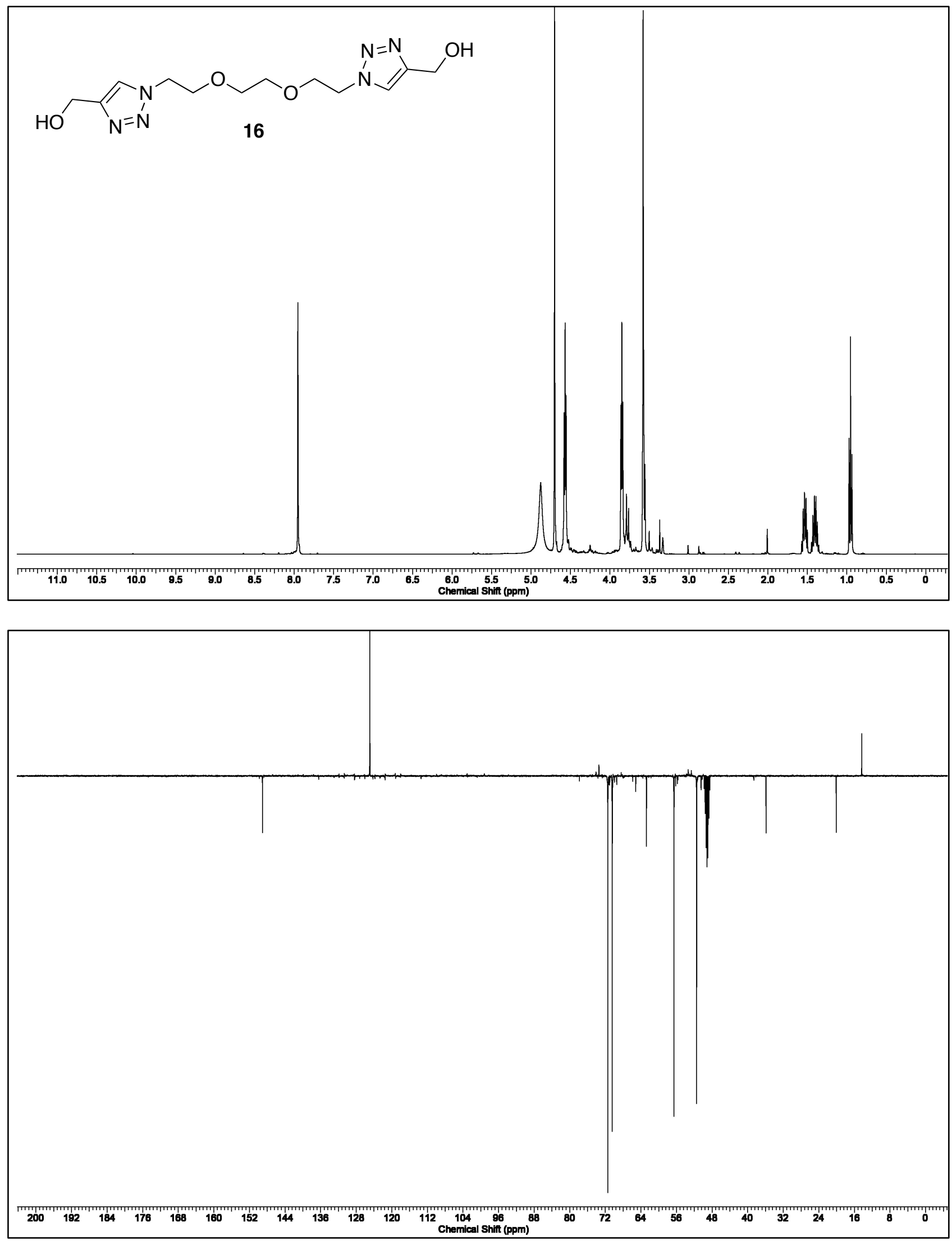

Figure S24. ${ }^{1} \mathrm{H} \& \mathrm{APT}-{ }^{13} \mathrm{C}$ NMR spectra of $\mathbf{1 6}$ 